\section{Nauplius}

The Journal of The

Brazilian Crustacean Society

e-ISSN 2358-2936

www.scielo.br/nau www.crustacea.org.br

\title{
Distribution and species diversity of freshwater crabs of the family Pseudothelphusidae in Colombia (Crustacea: Decapoda: Brachyura)
}

\author{
Martha R. Campos ${ }^{1}$ (D) orcid.org/0000-0001-5315-4933 \\ Diógenes Campos² (D) orcid.org/0000-0003-1990-3990
}
1 Universidad Nacional de Colombia, Instituto de Ciencias Naturales. Bogotá, Colombia.
MRC E-mail: mhrochad@unal.edu.co
2 Universidad Nacional de Colombia, Departamento de Física. Bogotá, Colombia. DC E-mail: dcamposr@unal.edu.co

ZOOBANK: http://zoobank.org/urn:1sid:zoobank.org:pub:3067787F-BB14-43D8859E-38D8057DE6EA

\section{ABSTRACT}

The study of Colombian freshwater crabs has advanced significantly, but species records are scattered across different museums and research institutions. Assuming that museum collections incorporate valuable information in estimating species diversity, a database was organized on the 94 known species of family Pseudothelphusidae, which includes 568 records from 1853 to 2019 in continental Colombia and Gorgona Island. The natural regions of Colombia (Amazonian, Andean, Caribbean, Orinoquian, Pacific) were further subdivided into 75 natural sub-regions according to habitat types used by freshwater crabs. Sub-regions were (i) ranked in terms of species richness, with respective species reported in each sub-region listed accordingly, and (ii) classified into a scale of five categories on the basis of a geometric biodiversity index combining species richness and Simpson index. Species accumulation curves are presented to estimate the current status of knowledge about the diversity of the Colombian pseudothelphusids. Although the available data are not standardized for an adequate assessment of relative abundance, since they are influenced by sampling efforts and natural sub-regions are of different sizes and characteristics, the present study may be useful in future ecological and biogeographic research, as well as for conservation purposes.

Corresponding Author Martha R. Campos mhrochad@unal.edu.co

\section{KeYWORDS}

Biodiversity index, ecological indicators, endemic and non-endemic species, Neotropical region, species richness 


\section{INTRODUCTION}

Colombia is considered the most species-rich country for freshwater crabs (109 species) in South America and the second worldwide after China (243 species) (Cumberlidge et al., 2011). Colombia has the highest endemism among the freshwater crabs of the family Pseudothelphusidae, with 86 species out of 94 endemic (91.5\%), whereas of the 15 recorded species of Trichodactylidae only one is endemic (6.7\%) (Campos, 2014).

Due to the direct relationship between environmental conditions and the distribution of freshwater crab populations, it is important to associate the species of the family Pseudothelphusidae with the five natural regions of continental Colombia (total land area approximately $1,141,748 \mathrm{~km}^{2}$ ), namely: the Caribbean, Andean, Pacific, Orinoquian and the Amazonian regions.

The characterization of Colombian natural regions is very broad (Alvarado, 2011). On the one hand, these areas differ from one another in many aspects, for example in the geographical size and in the physical, climatic, ecological, altitudinal and environmental conditions. On the other hand, natural regions connect with neighboring regions by transition zones, each one with their distinctive characteristics. In addition, many species are found in enclaves with particular ecological aspects. According to Alvarado's (2011) characterization, the natural regions of Colombia can be described as follows: (1) The Caribbean region comprises the area adjacent to the Caribbean Sea, which includes desert areas in La Guajira and rainforests in the Gulf of Uraba. The relief is characterized by being flat in its greatest extension. There are also some elevations such as the mountains of María, and the Sierra Nevada de Santa Marta, which is the highest in the region and the country. (2) The Andean region is made up of three major divisions formed by chains of high mountains (cordilleras), which are known as the Western, Central and Eastern cordilleras. This region covers approximately one third of the national territory, has a wide variety of altitude dependent climates, and is crossed by important rivers such as the Magdalena and Cauca, which run parallel to the mountains and flow into the Caribbean Sea. (3) The Pacific region has a high variety of climates and it is considered one of the wettest places on the planet. The hydrography is abundant and is formed by rivers such as the Atrato, Baudó and San Juan to the north of the region, and the Patía and Mira to the south of the region. (4) The Orinoquian region lies between the eastern mountain range, the Orinoco river, and the Arauca and Guaviare rivers. This region is formed by plains and savannahs and has some isolated mountain ranges with medium elevations. The main peak is the Serranía de la Macarena. (5) The Amazonian region in southern Colombia is mainly covered by tropical forests. It borders the Eastern cordillera along the western edge, in the north it includes the Guaviare and Vichada rivers and to the south it extends to the Putumayo and Amazon rivers. This region is formed by lowlands, with some undulations such as the Serrania de Chiribiquete. The weather is warm and very humid due to frequent rains.

The aim of this study is to do an assessment of the specific diversity of the Pseudothelphusidae freshwater crabs at the level of the selected 75 natural sub-regions and, even though the available data are not standardized for an adequate assessment of relative abundance (since they are influenced by sampling efforts and natural sub-regions are of different sizes and characteristics), to provide useful insights for future ecological and biogeographic research, as well as for conservation purposes.

\section{Material and Methods}

In order to assess the diversity of the pseudothelphusid crabs in the natural regions of Colombia, a database was first built listing the information available in the collections lots of Colombian pseudothelphusid crabs deposited in different museums and institutions around the world. Then, the sub-regions have been characterized in terms of their species richness by using a geometric index for measuring species diversity (Campos and Isaza, 2009).

\section{Database}

This study uses a database with 568 records of samples of Pseudothelphusidae crabs collected from 1853 to 2019 on continental Colombia and Gorgona Island. A total of 6,263 specimens corresponding to 
94 species, which are coded by consecutive numbers from 1 to 94, were included. The records are organized in a spreadsheet with 568 rows and 10 columns, in which the row contains the following ten fields of information: family (column 1), genus (2), species numerical code (3), species name (4), natural region (5), number of collected specimens (6), year sampled (7), institutional repository (8), natural sub-region collected from (9), and endemic status (10).

Data for the construction of the database were gathered from sixteen museums and research institutions, including species, type material, number of records and specimens (Tab. 1). Instituto de Ciencias Naturales, Museo de Historia Natural, Universidad Nacional de Colombia, Bogotá (ICN$\mathrm{MHN}$ ) is the greatest contributor to the database with $72.54 \%$ of the records and $86.91 \%$ of the number of specimens, while other museums and research institutions provide $27.46 \%$ and $13.09 \%$, respectively. The 94 Pseudothelphusidae species considered in this study include 4 subspecies of the Hypolobocera bouvieri (Rathbun, 1898) species-complex due to its presence in different sub-regions of Colombia.

Table 1. Museums and research institutions whose collections of the family Pseudothelphusidae have been used for the construction of the database. The last column includes the numbers of records (RE) and specimens (Specim). The abbreviations male (M) and female $(\mathrm{F})$ are used.

\begin{tabular}{|c|c|c|c|}
\hline Collection & Species name & Number of specimens and type material & RE/Specim \\
\hline \multirow[t]{23}{*}{ CRBMUV } & \multicolumn{2}{|c|}{ Museo de Biología Marina, Universidad del Valle, Cali } & $45 / 194$ \\
\hline & Hypolobocera alata & $\begin{array}{l}\text { 1M Holotype, 1F Paratype of Hypolobocera solimani Ramos-Tafur, } \\
2006 \text {, are junior synonym of } H \text {. alata }\end{array}$ & \\
\hline & Hypolobocera beieri & 95 & \\
\hline & Hypolobocera bouvieri angulata & 1 & \\
\hline & Hypolobocera bouvieri bouvieri & 7 & \\
\hline & Hypolobocera bouvieri monticola & 7 & \\
\hline & Hypolobocera buenaventurensis & $\begin{array}{l}\text { 11; } 1 \mathrm{M} \text { Holotype, } 1 \mathrm{~F} \text { Paratype of Hypolobocera olgaluciae Ramos- } \\
\text { Tafur and Ríos, } 2007 \text {, are junior synonym of } H \text {. buenaventurensis }\end{array}$ & \\
\hline & Hypolobocera cajambrensis & 5: 1M Holotype & \\
\hline & Hypolobocera chocoensis & $2 \mathrm{M}$ & \\
\hline & Hypolobocera dentata & 1M Holotype, 1F Paratype & \\
\hline & Hypolobocera gorgonensis & 9: 1M Holotype & \\
\hline & Hypolobocera lloroensis & 3 & \\
\hline & Hypolobocera. malaguena & 1M Holotype & \\
\hline & Hypolobocera martelathami & $\begin{array}{l}\text { 4; 1M Holotype of Hypolobocera merenbergiensis Prahl and Giraldo, } \\
1985 \text {, are junior synonym of H. martelathami) }\end{array}$ & \\
\hline & Hypolobocera meineli & 6: 1M Holotype, 1M Paratype & \\
\hline & Hypolobocera rotundilobata & 9 & \\
\hline & Lindacatalina orientalis & 3 & \\
\hline & Neostrengeria botti & 2 & \\
\hline & Neostrengeria guenteri & 2 & \\
\hline & Neostrengeria macropa & 5 & \\
\hline & Potamocarcinus colombiensis & 1M Holotype & \\
\hline & Phallangothelpusa dispar & 12 & \\
\hline & Strengeriana huilensis & 3 & \\
\hline \multirow[t]{2}{*}{ FMNH } & \multicolumn{2}{|c|}{ Field Museum of Natural History, Chicago } & $1 / 2$ \\
\hline & Moritschus caucasensis & 2 & \\
\hline \multirow[t]{2}{*}{ GM } & \multicolumn{2}{|r|}{ Genève Museum } & $1 / 4$ \\
\hline & Strengeriana restrepoi & 1M Holotype, 1M, 2F, 1 juvenile Paratypes & \\
\hline \multirow[t]{2}{*}{$\mathrm{IAvH}$} & \multicolumn{2}{|c|}{ Museum Alexander von Humboldt Institute, Villa de Leyva, Boyacá } & $1 / 3$ \\
\hline & Phallangothelpusa tangerina & 1M Holotype, 1M, 1F Paratypes & \\
\hline
\end{tabular}


Table 1. Cont.

\begin{tabular}{|c|c|c|c|}
\hline Collection & Species name & Number of specimens and type material & RE/Specim \\
\hline \multirow[t]{2}{*}{ ICN-MHN } & \multicolumn{2}{|c|}{ Instituto de Ciencias Naturales, Museo de Historia Natural, Universidad Nacional de Colombia, Bogotá } & $412 / 5443$ \\
\hline & \multicolumn{2}{|r|}{ 45M Holotypes, 293 Paratypes } & \\
\hline \multirow[t]{2}{*}{ INPA } & \multicolumn{2}{|c|}{ Instituto Nacional de Pesquisas da Amazônia, Manaus, Brazil } & $1 / 3$ \\
\hline & Moritschus caucasensis & 1M Holotype, 1M, 1F Paratypes & \\
\hline \multirow[t]{7}{*}{ IVIC } & \multicolumn{2}{|c|}{ Instituto Venezolano de Investigaciones Científicas, Caracas } & $6 / 38$ \\
\hline & Chaceus cesarensis & 1M Holotype, 1F Paratype & \\
\hline & Chaceus pearsei & 21 & \\
\hline & Fredius granulatus & 1M Paratype & \\
\hline & Martiana clausa & 5 & \\
\hline & Neostrengeria charalensis & 1 & \\
\hline & Neostrengeria sketi & 8 & \\
\hline \multirow[t]{3}{*}{ MB-UCV } & \multicolumn{2}{|c|}{ Universidad Central de Venezuela, Caracas } & $2 / 19$ \\
\hline & Chaceus nasutus & 1M Holotype & \\
\hline & Strengeriana tolimensis & 14M, 4F Paratypes & \\
\hline \multirow[t]{18}{*}{ MLS } & & Museo de La Salle, Bogotá & $29 / 193$ \\
\hline & Chaceus pearsei & 2 & \\
\hline & Hypolobocera beieri & 34 & \\
\hline & Hypolobocera bouvieri estenolobata & 1M Holotype & \\
\hline & Neostrengeria botti & 8 & \\
\hline & Neostrengeria boyacensis & 6: 1M Holotype, 1F Paratype & \\
\hline & Neostrengeria gilberti & 25 & \\
\hline & Neostrengeria guenteri & 12 & \\
\hline & Neostrengeria lasallei & 17: 1M Holotype, 2M Paratypes & \\
\hline & Neostrengeria libradensis & 1M Holotype & \\
\hline & Neostrengeria lindigiana & 30 & \\
\hline & Neostrengeria macropa & 13 & \\
\hline & Neostrengeria monterrodendoensis & 8 & \\
\hline & Neostrengeria niceforoi & 8 & \\
\hline & Phallangothelphusa dispar & 15 & \\
\hline & Strengeriana cajaensis & 3 & \\
\hline & Strengeriana casallasi & 1M Holotype, 2M, 3F Paratypes & \\
\hline & Strengeriana foresti & 1M Holotype & \\
\hline \multirow[t]{2}{*}{ MP } & \multicolumn{2}{|c|}{ Museum National d'Histoire Naturelle, Paris } & $1 / 4$ \\
\hline & Neostrengeria lindigiana & $1 \mathrm{M}, 2 \mathrm{~F}, 1$ juvenile Syntypes & \\
\hline \multirow[t]{10}{*}{ MUAICR } & \multicolumn{2}{|c|}{ Museo Universidad de Antioquia, Medellín, Colombia } & $16 / 110$ \\
\hline & Hypolobocera bouvieri monticola & 2 & \\
\hline & Hypolobocera chocoensis & 1 & \\
\hline & Neostrengeria macropa & 5 & \\
\hline & Phallangothelphusa dispar & 17 & \\
\hline & Strengeriana antioquensis & 21 & \\
\hline & Strengeriana bolivarensis & 5 & \\
\hline & Strengeriana flagellata & 9 & \\
\hline & Strengeriana fuhrmanni & 43 & \\
\hline & Strengeriana tolimensis & 5 & \\
\hline \multirow[t]{5}{*}{ NHM } & & atural History Museum, London & $5 / 26$ \\
\hline & Hypolobocera andagoensis & 1 & \\
\hline & Hypolobocera bouvieri monticola & 1M Holotype & \\
\hline & Hypolobocera chocoensis & 4M: 1M Holotype, 1M Paratype & \\
\hline & Strengeriana fuhrmanni & 20 Syntypes & \\
\hline
\end{tabular}


Table 1. Cont.

\begin{tabular}{|c|c|c|c|}
\hline Collection & Species name & Number of specimens and type material & RE/Specim \\
\hline \multirow[t]{12}{*}{ SMF } & \multicolumn{2}{|c|}{ Research Institute and Natural History Museum Senckenberg, Frankfurt } & $14 / 29$ \\
\hline & Chaceus nasutus & 1 & \\
\hline & Chaceus pearsei & 1 & \\
\hline & Neostrengeria botti & 12: $1 \mathrm{M}$ Holotype & \\
\hline & Neostrengeria charalensis & 1 & \\
\hline & Neostrengeria guenteri & 4 & \\
\hline & Neostrengeria lasallei & 1 & \\
\hline & Neostrengeria lindigiana & 1 & \\
\hline & Neostrengeria macropa & 3 & \\
\hline & Neostrengeria monterrodendoensis & 1M Paratype & \\
\hline & Neostrengeria niceforoi & 1 & \\
\hline & Strengeriana tolimensis & 1M Holotype, 2M, 1F Paratypes & \\
\hline \multirow[t]{16}{*}{ TU } & \multicolumn{2}{|c|}{ Museum of Natural History of Tulane University } & $19 / 66$ \\
\hline & Chaceus pearsei & 2 & \\
\hline & Hypolobocera beieri & 40 & \\
\hline & Hypolobocera bouvieri angulata & 1 & \\
\hline & Hypolobocera bouvieri bouvieri & 5 & \\
\hline & Hypolobocera gorgonensis & 1 & \\
\hline & Hypolobocera lloroensis & 1 & \\
\hline & Hypolobocera martelathami & 2 & \\
\hline & Hypolobocera noanamensis & 1M Holotype, 1F Paratype & \\
\hline & Hypolobocera rotundilobata & 2 & \\
\hline & Lindacatalina orientalis & 2 & \\
\hline & Lindacatalina sinuensis & 1M Holotype, 1F Paratype & \\
\hline & Lindacatalina sumacensis & 1 & \\
\hline & Moritschus altaquerensis & 1M, 1F Paratypes & \\
\hline & Phallangothelphusa dispar & 1 & \\
\hline & Strengeriana taironae & 2 & \\
\hline \multirow[t]{3}{*}{ UL } & \multicolumn{2}{|c|}{ Biology Institute, University of Ljubljana, Slovene } & $2 / 5$ \\
\hline & Neostrengeria niceforoi & 2 & \\
\hline & Neostrengeria sketi & 1M Holotype, 2F Paratypes & \\
\hline \multirow[t]{13}{*}{ USNM } & \multicolumn{2}{|c|}{ National Museum of Natural History, Smithsonian Institution, Washington, D.C. } & $13 / 124$ \\
\hline & Chaceus pearsei & 2: $1 \mathrm{M}$ Holotype & \\
\hline & Hypolobocera andagoensis & 63: 1M Holotype, 14M, 2F Paratypes & \\
\hline & Hypolobocera beieri & 6: 1M Holotype, 2M Paratypes & \\
\hline & Hypolobocera bouvieri angulata & 1M Holotype & \\
\hline & Hypolobocera cajambrensis & 1M Paratype & \\
\hline & Hypolobocera chocoensis & 36 & \\
\hline & Hypolobocera martelathami & 1M Holotype & \\
\hline & Hypolobocera mutisi & 1M Paratype & \\
\hline & Hypolobocera rotundilobata & $\begin{array}{l}\text { 7: } 1 \mathrm{M} \text { Holotype, } 5 \mathrm{M}, 1 \mathrm{~F} \text { Paratypes; } 1 \mathrm{M} \text { Holotype, } 5 \mathrm{M} \text { Paratypes of } \\
\text { Hypolobocera triangula Ramos-Tafur, 2006, are junior synonym of } H \text {. } \\
\text { rotundilobata }\end{array}$ & \\
\hline & Martiana clausa & 1M Holotype, 1M, 2F Paratypes & \\
\hline & Neostrengeria guenteri & 1M Holotype & \\
\hline & Neostrengeria perijaensis & 1M Paratype & \\
\hline TOTAL & & & $568 / 6263$ \\
\hline
\end{tabular}




\section{Natural sub-regions}

In this study we propose a characterization of the species of the family Pseudothelphusidae in a more localized level than that of the natural regions established in Alvarado (2011). For this, the hydrographic map of continental Colombia (IDEAM, 2013) was utilized to identify the habitat types from which freshwater crabs have been collected, such as watersheds, wetlands, streams, springs (Campos, 2014). Based on these criteria we have identified 75 sub-regions where the conditions for the presence of the crabs exist. As a microscope-like tool, the purpose of the division into 75 natural sub-regions is to advance the knowledge of the geographical distribution of the 94 species of the family Pseudothelphusidae by going from the macro-geographic level (five natural regions) to the micro-level (75 sub-regions). The location of the sub-regions is shown in Fig. 1, and the information for each of them is given in Tab. 2 .

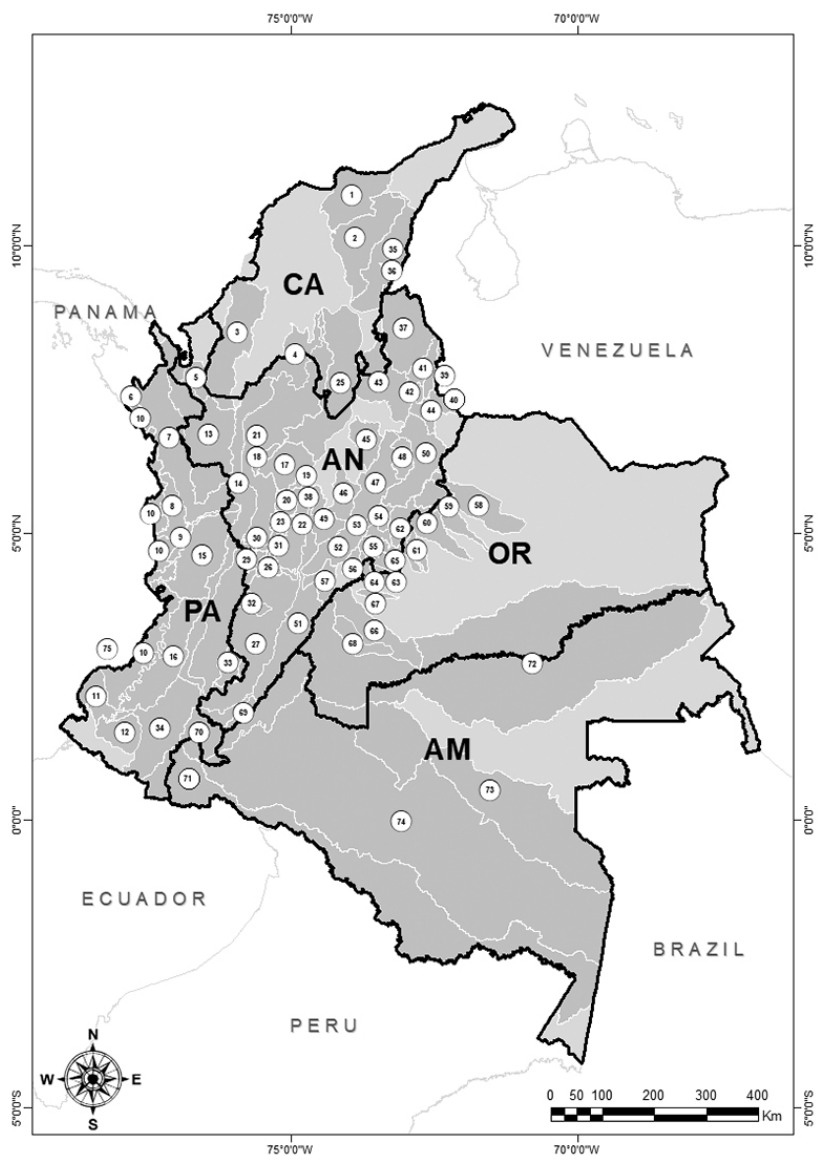

Figure 1. Natural regions of continental Colombia: Andean (AN), Amazonian (AM), Caribbean (CA), Orinoquian (OR) and Pacific (PA). In this study, 75 natural sub-regions are considered, including Gorgona island.
Each natural sub-region is identified by its full name, an acronym and a numerical code, which is useful not only for information processing but also to abbreviate the reference to it. For example, "Eastern Cordillera, Bogotá river" is referred to as "(52, CorBog)", according to the coding in Tab. 2.

The assignment of codes to species and subregions is intended to facilitate the management of information, i.e., the database can be accessed, managed, reorganized and updated without risk of changing information. During the analysis of the information, one of the authors (MRC) described three new species (Neostrengeria fernandezi Campos, 2017; Neostrengeria lassoi Campos, 2017; Phallangothelphusa tangerina Campos, Lasso and Arias, 2019), for which it was sufficient to assign them codes 92, 93, and 94.

The information in Tab. 2 is depicted in Fig. 2, in which the 94 species ( $y$-axis) are represented in terms of the sub-regions ( $x$-axis). Table 2 also lists the taxonomic authorities of all species cited in the main text and henceforward, for better fluency of reading, this information will be omitted in the text when the name of a species is mentioned for the first time.

\section{Species diversity index}

In order to quantify the diversity of pseudothelphusid freshwater crabs in Colombia and facilitate graphical data visualization, the species diversity index $B_{1}(S, r)$ proposed by Campos and Isaza (2009) was employed. This index has been applied in the characterization of ecological systems (Campos and Campos, 2012; 2017).

We are aware that: (i) The species diversity of freshwater crabs from Colombia has been affected over time, including the period from 1853 to 2019 in which the data was collected, by natural events and by the impact of anthropogenic actions (deforestation, environmental pollution, habitat fragmentation and climate change, among many others). (ii) The data obtained from the collections of museums and other research institutions has been gathered using different purposes and methodologies. Therefore, since they are influenced by the effort of the samplings, the available data do not reflect a standardized sampling of the number of species, nor of the relative abundance, and we only get a very rough description of the freshwater crabs of Colombia. However, it is noteworthy that 
records in databases have been successfully used by other authors as a surrogate of sampling effort (Soberón et al., 2007). (iii) The results provide baseline information that can be used for future observations and estimation of species diversity, and to assess whether diversity has been preserved, has declined or has increased.

In each one of the 75 sub-regions there is a $S$-species community, labeled as $\{1,2, \ldots, S\}$, that is represented in the database by $\left\{N_{1}, N_{2}, \ldots, N_{\mathrm{S}}\right\}$ specimens, for a total of $N=\sum_{m=1}^{S} N_{m}$ specimens associated with the subregion in consideration. Defining $p_{m}=N_{m} / N$, we find the probability distribution $p=\left\{p_{1}, p_{2}, \ldots, p_{s}\right\}$ that represents the relative abundance of species, which satisfies the normalization condition $\sum_{m=1}^{S} p_{m}=1$.

Denoting by $R E$ the number of records made in the sub-region under consideration, then $\left(N_{1}+N_{2}+\ldots\right.$ $\left.N_{S}\right) / R E$ is the average number of specimens captured in that sub-region (it is independent of the species to which they belong). Therefore, in order to standardize the number of specimens of the species among subregions, we substitute the set $\left\{N_{1}, N_{2}, \ldots, N_{s}\right\}$ by the values $\left\{N_{1} / R E, N_{2} / R E, \ldots, N_{S} / R E\right\}$, which add up
$N / R E$. The probability remains unchanged, since $p_{m}=\left(N_{m} / R E\right) /(N / R E)=N_{m} / N$, for $m=1,2, \ldots, S$.

A measure of homogeneity or concentration of any finite probability distribution, $p$, is the Simpson index D (Izsák and Papp, 2000),

$$
D=r^{2}:=\sum_{m=1}^{S}\left(p_{m}\right)^{2}
$$

that varies in the interval $0<D \leq 1$. Thus, low values of the index $D$ reveal homogeneity and high values indicate concentration. It is worth mentioning that the Simpson index $D$ describes the probability that two organisms drawn randomly and independently from a population belong to the same species. The above equation is related to a sphere of radius $r=\sqrt{D}$ in Euclidian space of $S$ dimensions, and $0<r \leq 1$. The species diversity index of order one (1) is given by the relation (Campos and Isaza, 2009)

$$
B_{1}(S, r)=\frac{\alpha_{1}(S)}{r}
$$

where $\alpha_{1}(S)$ is a monotonous increasing function of the number of species, $S$. The $B_{1}(S, r)$ index allows us to interpret changes in species diversity as

Table 2. Species of the family Pseudothelphusidae present in each one of the 75 sub-regions considered in this study. For each sub-region, it is specified: code and acronym (column 1), sub-region name and natural region to which it belongs. This is followed by (code, name) of the species recorded there (column 2), total numbers of records (RE) (column 3), number of species for each

\begin{tabular}{|c|c|c|c|c|}
\hline $\begin{array}{l}\text { Sub-region } \\
\text { Code and Acronym }\end{array}$ & $\begin{array}{l}\text { Name of sub-region and natural region. } \\
\text { Code and species name }\end{array}$ & RE & $\begin{array}{l}\text { Sp. } \\
(\mathbf{N})\end{array}$ & Spec. \\
\hline \multirow[t]{8}{*}{1, Ssm } & Sierra Nevada de Santa Marta, Caribbean natural region & & & \\
\hline & 4, Chaceus davidi Campos and Rodríguez, 1984 & & & 5 \\
\hline & 6, Chaceus nasutus Rodriguez, 1980 & & & 5 \\
\hline & 7, Chaceus pearsei (Rathbun, 1915) & & & 49 \\
\hline & $15 \Delta$, Hypolobocera bouvieri angulata (Rathbun, 1915) & & & 23 \\
\hline & 39, Martiana clausa Rodriguez, 1980 & & & 9 \\
\hline & 88, Strengeriana taironae Rodríguez and Campos, 1989 & & & 9 \\
\hline & & 32 & 6 & 100 \\
\hline \multirow[t]{5}{*}{ 2, CaribeCes } & Caribbean, Cesar river, Caribbean natural region & & & \\
\hline & 2, Chaceus cesarensis Rodríguez and Viloria, 1992 & & & 2 \\
\hline & 3, Chaceus curumanensis Campos and Valencia, 2004 & & & 2 \\
\hline & 5, Chaceus ibiricensis Campos and Valencia, 2004 & & & 86 \\
\hline & & 3 & 3 & 90 \\
\hline \multirow[t]{5}{*}{ 3, CaribeSi } & Caribbean, Sinú river, Caribbean natural region & & & \\
\hline & 32, Hypolobocera noanamensis Rodríguez, Campos and López, 2002 & & & 3 \\
\hline & 37, Lindacatalina sinuensis Rodríguez, Campos and López, 2002 & & & 25 \\
\hline & 69, Potamocarcinus pinzoni Campos, 2003 & & & 2 \\
\hline & & 6 & 3 & 30 \\
\hline
\end{tabular}
sub-region (column 4) and numbers of specimens (column 5). Symbol $\Delta$ indicates a non-endemic species. 
Table 2. Cont.

\begin{tabular}{|c|c|c|c|c|}
\hline $\begin{array}{l}\text { Sub-region } \\
\text { Code and Acronym }\end{array}$ & $\begin{array}{l}\text { Name of sub-region and natural region. } \\
\text { Code and species name }\end{array}$ & $\mathbf{R E}$ & $\begin{array}{l}\text { Sp. } \\
(\mathbf{N})\end{array}$ & Spec. \\
\hline \multirow[t]{2}{*}{ 4, CaribeCau } & Caribbean, Cauca river, Caribbean natural region & & & \\
\hline & & 1 & 1 & 2 \\
\hline \multirow[t]{2}{*}{ 5, CbioLeo } & Biogeographic Chocó, León river, Pacific natural region & & & \\
\hline & & 1 & 1 & 1 \\
\hline
\end{tabular}

6, CbioJur Biogeographic Chocó, Juradó river, Pacific natural region

68, Potamocarcinus colombiensis Prahl and Ramos, 1987

\begin{tabular}{|c|c|c|c|c|}
\hline & & 1 & 1 & 1 \\
\hline \multirow[t]{7}{*}{ 7, CbioAt } & Biogeographic Chocó, Atrato river, Pacific natural region & & & \\
\hline & 1, Colombiathelphusa culmarcuata Campos and Magalhães, 2014 & & & 12 \\
\hline & $8 \Delta$, Eidocamptophallus chacei (Pretzmann, 1967) & & & 1 \\
\hline & 21, Hypolobocera chocoensis Rodriguez, 1980 & & & 2 \\
\hline & 26, Hypolobocera lloroensis Campos, 1989 & & & 11 \\
\hline & 69, Potamocarcinus pinzoni Campos, 2003 & & & 15 \\
\hline & & 13 & 6 & 51 \\
\hline
\end{tabular}

8, CbioBau Biogeographic Chocó, Baudó river, Pacific natural region

21, Hypolobocera chocoensis Rodriguez, 1980

32, Hypolobocera noanamensis Rodríguez, Campos and López, 2002

69, Potamocarcinus pinzoni Campos, 2003

$6 \quad 3 \quad 19$

9, CbioSJ

Biogeographic Chocó, San Juan river, Pacific natural region

11, Hypolobocera alata Campos, 1989

12, Hypolobocera andagoensis (Pretzmann, 1965)

21, Hypolobocera chocoensis Rodríguez, 1980

26, Hypolobocera lloroensis Campos, 1989

32, Hypolobocera noanamensis Rodriguez, Campos and López, 2002

12

5

Biogeographic Chocó, Pacific, Pacific natural region

19, Hypolobocera buenaventurensis (Rathbun, 1905)

20, Hypolobocera cajambrensis Prahl, 1988

26, Hypolobocera lloroensis Campos, 1989

27, Hypolobocera malaguena Prahl, 1988

29, Hypolobocera meineli Prahl, 1988

31, Hypolobocera mutisi Prahl, 1988

11, CbioPat

Biogeographic Chocó, Patía river, Pacific natural region

13, Hypolobocera barbacensis Campos, Magalhães and Rodríguez, 2003

29, Hypolobocera meineli Prahl, 1988

40, Moritschus altaquerensis Rodríguez, Campos and López, 2002 
Table 2. Cont.

\begin{tabular}{|c|c|c|c|c|}
\hline $\begin{array}{l}\text { Sub-region } \\
\text { Code and Acronym }\end{array}$ & $\begin{array}{l}\text { Name of sub-region and natural region. } \\
\text { Code and species name }\end{array}$ & $\mathbf{R E}$ & $\begin{array}{l}\text { Sp. } \\
(\mathbf{N})\end{array}$ & Spec. \\
\hline \multirow[t]{4}{*}{ 13, CocAt } & Western cordillera, Atrato river, Andean natural region & & & \\
\hline & 23, Hypolobocera emberarum Campos and Rodríguez, 1995 & & & 30 \\
\hline & 26, Hypolobocera lloroensis Campos, 1989 & & & 28 \\
\hline & & 10 & 4 & 69 \\
\hline
\end{tabular}

\begin{tabular}{|c|c|c|c|c|}
\hline \multirow[t]{7}{*}{ 14, CocCau } & \multicolumn{4}{|l|}{ Western cordillera, Cauca river, Andean natural region } \\
\hline & \multicolumn{3}{|l|}{ 14, Hypolobocera beieri Pretzmann, 1968} & 118 \\
\hline & \multicolumn{3}{|l|}{ 17, Hypolobocera bouvieri monticola (Zimmer, 1912) } & 3 \\
\hline & \multicolumn{3}{|l|}{ 22, Hypolobocera dentata Prahl, 1987} & 2 \\
\hline & \multicolumn{3}{|l|}{ 41, Moritschus caucasensis Campos, Magalhães and Rodríguez, 2003} & 5 \\
\hline & \multicolumn{3}{|l|}{ 86, Strengeriana restrepoi Rodriguez, 1980} & 2 \\
\hline & & 16 & 5 & 130 \\
\hline \multirow[t]{9}{*}{$15, \mathrm{CocSJ}$} & \multicolumn{4}{|l|}{ Western cordillera, San Juan river, Andean natural region } \\
\hline & \multicolumn{3}{|l|}{ 11, Hypolobocera alata Campos, 1989} & 8 \\
\hline & \multicolumn{3}{|l|}{ 14, Hypolobocera beieri Pretzmann, 1968} & 3 \\
\hline & \multicolumn{3}{|l|}{ 17, Hypolobocera bouvieri monticola (Zimmer, 1912) } & 151 \\
\hline & \multicolumn{3}{|l|}{ 21, Hypolobocera chocoensis Rodriguez, 1980} & 2 \\
\hline & \multicolumn{3}{|l|}{ 33, Hypolobocera rotundilobata Rodríguez, 1994} & 20 \\
\hline & \multicolumn{3}{|l|}{ 34, Hypolobocera velezi Campos, 2003} & 7 \\
\hline & \multicolumn{3}{|l|}{ 87, Strengeriana risaraldensis Rodríguez and Campos, 1989} & 109 \\
\hline & & 18 & 8 & 305 \\
\hline \multirow[t]{4}{*}{ 16, CocPac } & \multicolumn{4}{|l|}{ Western cordillera, Pacific, Andean natural region } \\
\hline & \multicolumn{3}{|l|}{ 14, Hypolobocera beieri Pretzmann, 1968} & 24 \\
\hline & \multicolumn{3}{|l|}{ 19, Hypolobocera buenaventurensis (Rathbun, 1905) } & 11 \\
\hline & & 12 & 2 & 35 \\
\hline \multirow[t]{2}{*}{ 17, CceNare } & \multicolumn{4}{|l|}{ Central cordillera, Nare river, Andean natural region } \\
\hline & \multicolumn{3}{|l|}{ 17, Hypolobocera bouvieri monticola (Zimmer, 1912) } & 1 \\
\hline
\end{tabular}

18, CceMed $\quad$ Central cordillera, Medellín river, Andean natural regi
16, Hypolobocera bouvieri bouvieri (Rathbun, 1898)
17, Hypolobocera bouvieri monticola (Zimmer, 1912)
75, Strengeriana antioquensis Prahl, 1987

$\begin{array}{cc}4 \\ 4 \\ 10 & 27 \\ & 35\end{array}$

19, CceCoco Central cordillera, Cocorná river, Andean natural region

80, Strengeriana flagellata Campos and Rodríguez, 1993

Central cordillera, La Miel river, Andean natural region

17, Hypolobocera bouvieri monticola (Zimmer, 1912)

70, Phallangothelphusa dispar (Zimmer, 1912)

81, Strengeriana florenciae Campos, 1995

85, Strengeriana maniformis Campos and Rodríguez, 1993

21, CceCau

Central cordillera, Cauca river, Andean natural region 
Table 2. Cont.

\begin{tabular}{|c|c|c|c|c|}
\hline $\begin{array}{l}\text { Sub-region } \\
\text { Code and Acronym }\end{array}$ & $\begin{array}{l}\text { Name of sub-region and natural region. } \\
\text { Code and species name }\end{array}$ & $\mathbf{R E}$ & $\begin{array}{l}\text { Sp. } \\
(\mathbf{N})\end{array}$ & Spec. \\
\hline & 76, Strengeriana bolivarensis Rodríguez and Campos, 1989 & & & 8 \\
\hline & 83, Strengeriana fuhrmanni (Zimmer, 1912) & & & 75 \\
\hline & 86, Strengeriana restrepoi Rodriguez, 1980 & & & 4 \\
\hline & & 21 & 4 & 101 \\
\hline \multirow[t]{4}{*}{ 22, CceGual } & Central cordillera, Gualí river, Andean natural region & & & \\
\hline & 70, Phallangothelphusa dispar (Zimmer, 1912) & & & 2 \\
\hline & 85, Strengeriana maniformis Campos and Rodríguez, 1993 & & & 1 \\
\hline & & 2 & 2 & 3 \\
\hline \multirow[t]{4}{*}{ 23, CceGuar } & Central cordillera, Guarinó river, Andean natural region & & & \\
\hline & 82, Strengeriana foresti Rodríguez, 1980 & & & 1 \\
\hline & 85, Strengeriana maniformis Campos and Rodríguez, 1993 & & & 1 \\
\hline & & 2 & 2 & 2 \\
\hline \multirow[t]{3}{*}{ 24, CceBar } & Central cordillera, Barbas river, Andean natural region & & & \\
\hline & 86, Strengeriana restrepoi Rodriguez, 1980 & & & 2 \\
\hline & & 1 & 1 & 2 \\
\hline \multirow[t]{6}{*}{ 25, CceMa } & Central cordillera, Magdalena river, Andean natural region & & & \\
\hline & 17, Hypolobocera bouvieri monticola (Zimmer, 1912) & & & 1 \\
\hline & 28, Hypolobocera martelathami (Pretzmann, 1965) & & & 48 \\
\hline & 70, Phallangothelphusa dispar (Zimmer, 1912) & & & 3 \\
\hline & 84, Strengeriana huilensis Rodríguez and Campos, 1989 & & & 3 \\
\hline & & 4 & 4 & 55 \\
\hline \multirow[t]{5}{*}{ 26, CceCoe } & Central cordillera, Coello river, Andean natural region & & & \\
\hline & 77, Strengeriana cajaensis Campos and Rodríguez, 1993 & & & 2 \\
\hline & 85, Strengeriana maniformis Campos and Rodríguez, 1993 & & & 3 \\
\hline & 89, Strengeriana tolimensis Rodriguez and Diaz, 1981 & & & 24 \\
\hline & & 5 & 3 & 29 \\
\hline \multirow[t]{3}{*}{ 27, CceBog } & Central cordillera, Bogotá river, Andean natural region & & & \\
\hline & 70, Phallangothelphusa dispar (Zimmer, 1912) & & & 5 \\
\hline & & 2 & 1 & 5 \\
\hline \multirow[t]{5}{*}{ 28, CceAzu } & Central cordillera, Azufrado river, Andean natural region & & & \\
\hline & 77, Strengeriana cajaensis Campos and Rodríguez, 1993 & & & 35 \\
\hline & 78, Strengeriana casallasi Campos, 1999 & & & 7 \\
\hline & 90, Strengeriana villaensis Campos and Pedraza, 2006 & & & 4 \\
\hline & & 8 & 3 & 46 \\
\hline \multirow[t]{3}{*}{ 29, CceQui } & Central cordillera, Quindio river, Andean natural region & & & \\
\hline & 89, Strengeriana tolimensis Rodriguez and Diaz, 1981 & & & 3 \\
\hline & & 1 & 1 & 3 \\
\hline \multirow[t]{3}{*}{ 30, CceOtun } & Central cordillera, Otún river, Andean natural region & & & \\
\hline & 86, Strengeriana restrepoi Rodriguez, 1980 & & & 24 \\
\hline & & 2 & 1 & 24 \\
\hline \multirow[t]{3}{*}{ 31, CceCom } & Central cordillera, Combeima river, Andean natural region & & & \\
\hline & 89, Strengeriana tolimensis Rodríguez and Díaz, 1981 & & & 1 \\
\hline & & 1 & 1 & 1 \\
\hline \multirow[t]{4}{*}{ 32, CceAmo } & Central cordillera, Amoyá river, Andean natural region & & & \\
\hline & 17, Hypolobocera bouvieri monticola (Zimmer, 1912) & & & 94 \\
\hline & 70, Phallangothelphusa dispar (Zimmer, 1912) & & & 2 \\
\hline & 79, Strengeriana chaparralensis Campos and Rodríguez, 1984 & & & 50 \\
\hline
\end{tabular}


Table 2. Cont.

\begin{tabular}{|c|c|c|c|c|}
\hline $\begin{array}{l}\text { Sub-region } \\
\text { Code and Acronym }\end{array}$ & $\begin{array}{l}\text { Name of sub-region and natural region. } \\
\text { Code and species name }\end{array}$ & $\mathbf{R E}$ & $\begin{array}{l}\text { Sp. } \\
(\mathbf{N})\end{array}$ & Spec \\
\hline & 89, Strengeriana tolimensis Rodriguez and Diaz, 1981 & & & 10 \\
\hline & & 20 & 4 & 156 \\
\hline \multirow[t]{4}{*}{ 33, CcePaez } & Central cordillera, Páez river, Andean natural region & & & \\
\hline & 28, Hypolobocera martelathami (Pretzmann, 1965) & & & 14 \\
\hline & $36 \Delta$, Lindacatalina orientalis (Pretzmann, 1968) & & & 7 \\
\hline & & 5 & 2 & 21 \\
\hline \multirow[t]{4}{*}{ 34, CcePat } & Central cordillera, Patía river, Andean natural region & & & \\
\hline & 14, Hypolobocera beieri Pretzmann, 1968 & & & 44 \\
\hline & $36 \Delta$, Lindacatalina orientalis (Pretzmann, 1968) & & & 19 \\
\hline & & 8 & 2 & 63 \\
\hline \multirow[t]{3}{*}{35 , CorCes } & Eastern cordillera, Cesar river, Andean natural region & & & \\
\hline & $15 \Delta$, Hypolobocera bouvieri angulata (Rathbun, 1915 ) & & & 1 \\
\hline & & 1 & 1 & 1 \\
\hline \multirow[t]{4}{*}{ 36, CorTuc } & Eastern cordillera, Tucuy river, Andean natural region & & & \\
\hline & $15 \Delta$, Hypolobocera bouvieri angulata (Rathbun, 1915) & & & 44 \\
\hline & 63, Neostrengeria perijaensis Campos and Lemaitre, 1998 & & & 52 \\
\hline & & 3 & 2 & 96 \\
\hline \multirow[t]{3}{*}{ 37, CorCat } & Eastern cordillera, Catatumbo river, Andean natural region & & & \\
\hline & 15, Hypolobocera bouvieri angulata (Rathbun, 1915) & & & 6 \\
\hline & & 2 & 1 & 6 \\
\hline \multirow[t]{4}{*}{ 38, MmeMa } & Middle Magdalena, Magdalena river, Andean natural region & & & \\
\hline & 15, Hypolobocera bouvieri angulata (Rathbun, 1915) & & & 1 \\
\hline & 72, Phallangothelphusa magdalenensis Campos, 1998 & & & 65 \\
\hline & & 5 & 2 & 66 \\
\hline \multirow[t]{3}{*}{ 39, CorPam } & Eastern cordillera, Pamplonita river, Andean natural region & & & \\
\hline & 65, Neostrengeria tencalanensis Campos, 1992 & & & 148 \\
\hline & & 2 & 1 & 148 \\
\hline \multirow[t]{4}{*}{ 40, CorTach } & Eastern cordillera, Táchira river, Andean natural region & & & \\
\hline & 44, Neostrengeria appressa Campos, 1992 & & & 149 \\
\hline & $67 \Delta$, Orthothelphusa holthuisi (Rodríguez, 1967) & & & 33 \\
\hline & & 3 & 2 & 182 \\
\hline \multirow[t]{3}{*}{ 41, CorZu } & Eastern cordillera, Zulia river, Andean natural region & & & \\
\hline & 57, Neostrengeria lobulata Campos, 1992 & & & 18 \\
\hline & & 2 & 1 & 18 \\
\hline \multirow[t]{3}{*}{ 42, CorCuc } & Eastern cordillera, Cucutilla river, Andean natural region & & & \\
\hline & 16, Hypolobocera bouvieri bouvieri (Rathbun, 1898) & & & 3 \\
\hline & & 1 & 1 & 3 \\
\hline \multirow[t]{6}{*}{ 43, CorLebr } & Eastern cordillera, Lebrija river, Andean natural region & & & \\
\hline & 15, Hypolobocera bouvieri angulata (Rathbun, 1915) & & & 1 \\
\hline & 16, Hypolobocera bouvieri bouvieri (Rathbun, 1898) & & & 133 \\
\hline & 66, Neostrengeria tonensis Campos, 1992 & & & 155 \\
\hline & 73, Phallangothelphusa martensis Cardona and Campos, 2012 & & & 10 \\
\hline & & 11 & 4 & 299 \\
\hline \multirow[t]{3}{*}{ 44, CorMar } & Eastern cordillera, Margua river, Andean natural region & & & \\
\hline & 91, Neostrengeria libradensis Rodriguez, 1980 & & & 1 \\
\hline & & 1 & 1 & 1 \\
\hline
\end{tabular}


Table 2. Cont.

\begin{tabular}{|c|c|c|c|c|}
\hline $\begin{array}{l}\text { Sub-region } \\
\text { Code and Acronym }\end{array}$ & $\begin{array}{l}\text { Name of sub-region and natural region. } \\
\text { Code and species name }\end{array}$ & $\mathbf{R E}$ & $\begin{array}{l}\text { Sp. } \\
(\mathbf{N})\end{array}$ & Spec. \\
\hline \multirow[t]{5}{*}{ 45, CorOpon } & Eastern cordillera, Opón river, Andean natural region & & & \\
\hline & 16, Hypolobocera bouvieri bouvieri (Rathbun, 1898) & & & 4 \\
\hline & 64, Neostrengeria sketi Rodríguez, 1985 & & & 16 \\
\hline & 94, Phallangothelphusa tangerina Campos, Lasso and Arias, 2019 & & & 15 \\
\hline & & 7 & 3 & 35 \\
\hline \multirow[t]{9}{*}{ 46, CorMin } & Eastern cordillera, Minero river, Andean natural region & & & \\
\hline & 16, Hypolobocera bouvieri bouvieri (Rathbun, 1898) & & & 31 \\
\hline & 45, Neostrengeria aspera Campos, 1992 & & & 2 \\
\hline & 48, Neostrengeria botti Rodríguez and Türkay, 1978 & & & 151 \\
\hline & 52, Neostrengeria gilberti Campos, 1992 & & & 34 \\
\hline & 55, Neostrengeria lemaitrei Campos, 2004 & & & 10 \\
\hline & 92, Neostrengeria fernandezi Campos, 2017 & & & 4 \\
\hline & 72, Phallangothelphusa magdalenensis Campos, 1998 & & & 66 \\
\hline & & 12 & 7 & 298 \\
\hline \multirow[t]{5}{*}{ 47, CorSua } & Eastern cordillera, Suárez river, Andean natural region & & & \\
\hline & 16, Hypolobocera bouvieri bouvieri (Rathbun, 1898) & & & 29 \\
\hline & 51, Neostrengeria charalensis Campos and Rodríguez, 1985 & & & 283 \\
\hline & 62, Neostrengeria niceforoi (Schmitt, 1969) & & & 80 \\
\hline & & 24 & 3 & 392 \\
\hline
\end{tabular}

48, CorFon

Eastern cordillera, Fonce river, Andean natural region

51, Neostrengeria charalensis Campos and Rodríguez, $1985 \quad 18$

62, Neostrengeria niceforoi (Schmitt, 1969)

$10-105$

49, CorNeg1 Eastern cordillera, Negro river 1, Andean natural region

16, Hypolobocera bouvieri bouvieri (Rathbun, 1898)

45, Neostrengeria aspera Campos, 1992

48, Neostrengeria botti Rodríguez and Türkay, 1978

52, Neostrengeria gilberti Campos, $1992 \quad 4$

56, Neostrengeria lindigiana (Rathbun, 1897) 15

70, Phallangothelphusa dispar (Zimmer, 1912)

72, Phallangothelphusa magdalenensis Campos, 1998

$\begin{array}{llll}27 & 7 & 385\end{array}$

50, CorChi Eastern cordillera, Chicamocha river, Andean natural region

16, Hypolobocera bouvieri bouvieri (Rathbun, 1898)

49, Neostrengeria boyacensis Rodriguez, 1980

$8 \quad 2 \quad 188$

51, CorMa $\quad$ Eastern cordillera, Magdalena river, Andean natural region

16, Hypolobocera bouvieri bouvieri (Rathbun, 1898) 61

18, Hypolobocera bouvieri estenolobata Rodriguez, $1980 \quad 18$

48, Neostrengeria botti Rodríguez and Türkay, 1978

93, Neostrengeria lassoi Campos, $2017 \quad 4$

70, Phallangothelphusa dispar (Zimmer, 1912) 12

84, Strengeriana huilensis Rodríguez and Campos, 1989

52, CorBog $\quad$ Eastern cordillera, Bogotá river, Andean natural region $\quad 16 \quad 6 \quad 13$

16, Hypolobocera bouvieri bouvieri (Rathbun, 1898) 26

47, Neostrengeria binderi Campos, $2000 \quad 3$

48, Neostrengeria botti Rodríguez and Türkay, 1978 
Table 2. Cont.

\begin{tabular}{|c|c|c|c|c|}
\hline $\begin{array}{l}\text { Sub-region } \\
\text { Code and Acronym }\end{array}$ & $\begin{array}{l}\text { Name of sub-region and natural region. } \\
\text { Code and species name }\end{array}$ & $\mathbf{R E}$ & $\begin{array}{l}\text { Sp. } \\
(\mathbf{N})\end{array}$ & Spec. \\
\hline & 52, Neostrengeria gilberti Campos, 1992 & & & 10 \\
\hline & 56, Neostrengeria lindigiana (Rathbun, 1897) & & & 83 \\
\hline & 59, Neostrengeria macropa (H. Milne Edwards, 1853) & & & 168 \\
\hline & 70, Phallangothelphusa dispar (Zimmer, 1912) & & & 107 \\
\hline & & 55 & 7 & 450 \\
\hline \multirow[t]{4}{*}{ 53, CorBat } & Eastern cordillera, Batá river, Andean natural region & & & \\
\hline & 46, Neostrengeria bataensis Campos and Pedraza, 2008 & & & 1 \\
\hline & 54, Neostrengeria lasallei Rodríguez, 1980 & & & 57 \\
\hline & & 7 & 2 & 58 \\
\hline \multirow[t]{4}{*}{ 54, CorGar } & Eastern cordillera, Garagoa river, Andean natural region & & & \\
\hline & 49, Neostrengeria boyacensis Rodríguez, 1980 & & & 97 \\
\hline & 54, Neostrengeria lasallei Rodriguez, 1980 & & & 192 \\
\hline & & 7 & 2 & 289 \\
\hline \multirow[t]{4}{*}{ 55, CorGua } & Eastern cordillera, Guavio river, Andean natural region & & & \\
\hline & 46, Neostrengeria bataensis Campos and Pedraza, 2008 & & & 24 \\
\hline & 54, Neostrengeria lasallei Rodriguez, 1980 & & & 41 \\
\hline & & 4 & 2 & 65 \\
\hline \multirow[t]{5}{*}{56, CorNeg2 } & Eastern cordillera, Negro river 2, Andean natural region & & & \\
\hline & 53, Neostrengeria guenteri (Pretzmann, 1965) & & & 82 \\
\hline & 56, Neostrengeria lindigiana (Rathbun, 1897) & & & 1 \\
\hline & 60, Neostrengeria monterrodendoensis (Bott, 1967) & & & 143 \\
\hline & & 10 & 3 & 226 \\
\hline \multirow[t]{7}{*}{ 57, CorSum } & Eastern cordillera, Sumapaz river, Andean natural region & & & \\
\hline & 16, Hypolobocera bouvieri bouvieri (Rathbun) & & & 6 \\
\hline & 47, Neostrengeria binderi Campos, 2000 & & & 1 \\
\hline & 56, Neostrengeria lindigiana (Rathbun, 1897) & & & 42 \\
\hline & 70, Phallangothelphusa dispar (Zimmer, 1912) & & & 24 \\
\hline & 77, Strengeriana cajaensis Campos and Rodríguez, 1993 & & & 21 \\
\hline & & 18 & 5 & 94 \\
\hline \multirow[t]{3}{*}{58, OriPau } & Orinoquian, Pauto river, Orinoquian natural region & & & \\
\hline & 9, Eudaniela casanarensis (Campos, 2001) & & & 26 \\
\hline & & 2 & 1 & 26 \\
\hline \multirow[t]{4}{*}{59 , OriCra } & Orinoquian, Cravo Sur river, Orinoquian natural region & & & \\
\hline & 9, Eudaniela casanarensis (Campos, 2001) & & & 70 \\
\hline & 50, Neostrengeria celioi Campos and Pedraza, 2008 & & & 5 \\
\hline & & 4 & 2 & 75 \\
\hline \multirow[t]{5}{*}{60, OriCus } & Orinoquian, Cusiana river, Orinoquian natural region & & & \\
\hline & 9, Eudaniela casanarensis (Campos, 2001) & & & 47 \\
\hline & 46, Neostrengeria bataensis Campos and Pedraza, 2008 & & & 14 \\
\hline & 50, Neostrengeria celioi Campos and Pedraza, 2008 & & & 36 \\
\hline & & 6 & 3 & 97 \\
\hline \multirow[t]{4}{*}{ 61, OriTua } & Orinoquian, Túa river, Orinoquian natural region & & & \\
\hline & 9, Eudaniela casanarensis (Campos, 2001) & & & 10 \\
\hline & 46, Neostrengeria bataensis Campos and Pedraza, 2008 & & & 1 \\
\hline & & 2 & 2 & 11 \\
\hline \multirow[t]{3}{*}{ 62, CorUpia } & Eastern cordillera, Upía river, Andean natural region & & & \\
\hline & 49, Neostrengeria boyacensis Rodriguez, 1980 & & & 4 \\
\hline & & 1 & 1 & 4 \\
\hline
\end{tabular}


Table 2. Cont.

\begin{tabular}{|c|c|c|c|c|}
\hline $\begin{array}{l}\text { Sub-region } \\
\text { Code and Acronym }\end{array}$ & $\begin{array}{l}\text { Name of sub-region and natural region. } \\
\text { Code and species name }\end{array}$ & $\mathbf{R E}$ & $\begin{array}{l}\text { Sp. } \\
(\mathbf{N})\end{array}$ & Spec. \\
\hline \multirow[t]{3}{*}{ 63, CorGuac } & Eastern cordillera, Guacavía river, Andean natural region & & & \\
\hline & 53, Neostrengeria guenteri (Pretzmann, 1965) & & & 126 \\
\hline & & 4 & 1 & 126 \\
\hline \multirow[t]{4}{*}{ 64, CorGuat } & Eastern cordillera, Guatiquía river, Andean natural region & & & \\
\hline & 46, Neostrengeria bataensis Campos and Pedraza, 2008 & & & 1 \\
\hline & 53, Neostrengeria guenteri (Pretzmann, 1965) & & & 297 \\
\hline & & 16 & 2 & 298 \\
\hline \multirow[t]{4}{*}{65 , OriHum } & Orinoquian, Humea river, Orinoquian natural region & & & \\
\hline & 43, Neostrengeria alexae Campos, $2010 \mathrm{~b}$ & & & 8 \\
\hline & 46, Neostrengeria bataensis Campos and Pedraza, 2008 & & & 18 \\
\hline & & 2 & 2 & 26 \\
\hline \multirow[t]{3}{*}{ 66, OriAri } & Orinoquian, Ariari river, Orinoquian natural region & & & \\
\hline & 53, Neostrengeria guenteri (Pretzmann, 1965) & & & 43 \\
\hline & & 2 & 1 & 43 \\
\hline \multirow[t]{4}{*}{ 67, OriAca } & Orinoquian, Acacías river, Orinoquian natural region & & & \\
\hline & 53, Neostrengeria guenteri (Pretzmann, 1965) & & & 24 \\
\hline & 61, Neostrengeria natashae Campos, 2011 & & & 2 \\
\hline & & 5 & 2 & 26 \\
\hline \multirow[t]{3}{*}{68 , OriGue } & Orinoquian, Güejar river, Orinoquian natural region & & & \\
\hline & 58, Neostrengeria macarenae Campos, 1992 & & & 1 \\
\hline & & 1 & 1 & 1 \\
\hline \multirow[t]{3}{*}{ 69, CorSuaza } & Eastern cordillera, Suaza river, Andean natural region & & & \\
\hline & 28, Hypolobocera martelathami (Pretzmann, 1965) & & & 23 \\
\hline & & 1 & 1 & 23 \\
\hline \multirow[t]{4}{*}{ 70, CorCa } & Eastern cordillera, Caquetá river, Andean natural region & & & \\
\hline & 25, Hypolobocera kamsarum Campos and Rodríguez, 1995 & & & 5 \\
\hline & $35 \Delta$, Lindacatalina latipenis (Pretzmann, 1968) & & & 9 \\
\hline & & 3 & 2 & 14 \\
\hline \multirow[t]{3}{*}{ 71, AmazoPu } & Amazonian, Putumayo river, Amazonian natural region & & & \\
\hline & 38 $\Delta$, Lindacatalina sumacensis Rodríguez and Sternberg, 1998 & & & 4 \\
\hline & & 3 & 1 & 4 \\
\hline \multirow[t]{3}{*}{ 72, AmazoGua } & Amazonian, Guaviare river, Amazonian natural region & & & \\
\hline & 74ム, Prionothelphusa eliasi Rodriguez, 1980 & & & 2 \\
\hline & & 2 & 1 & 2 \\
\hline \multirow[t]{4}{*}{ 73, AmazoApa } & Amazonian, Apaporis river, Amazonian natural region & & & \\
\hline & 10, Fredius granulatus Rodríguez and Campos, 1998 & & & 12 \\
\hline & 74ム, Prionothelphusa eliasi Rodriguez, 1980 & & & 3 \\
\hline & & 4 & 2 & 15 \\
\hline \multirow[t]{3}{*}{ 74, AmazoCa } & Amazonian, Caquetá river, Amazonian natural region & & & \\
\hline & $74 \Delta$, Prionothelphusa eliasi Rodríguez, 1980 & & & 3 \\
\hline & & 1 & 1 & 3 \\
\hline \multirow[t]{3}{*}{ 75, Gor } & Gorgona Island, Pacific natural region & & & \\
\hline & 24A, Hypolobocera gorgonensis Prahl, 1983 & & & 13 \\
\hline & & 6 & 1 & 13 \\
\hline
\end{tabular}




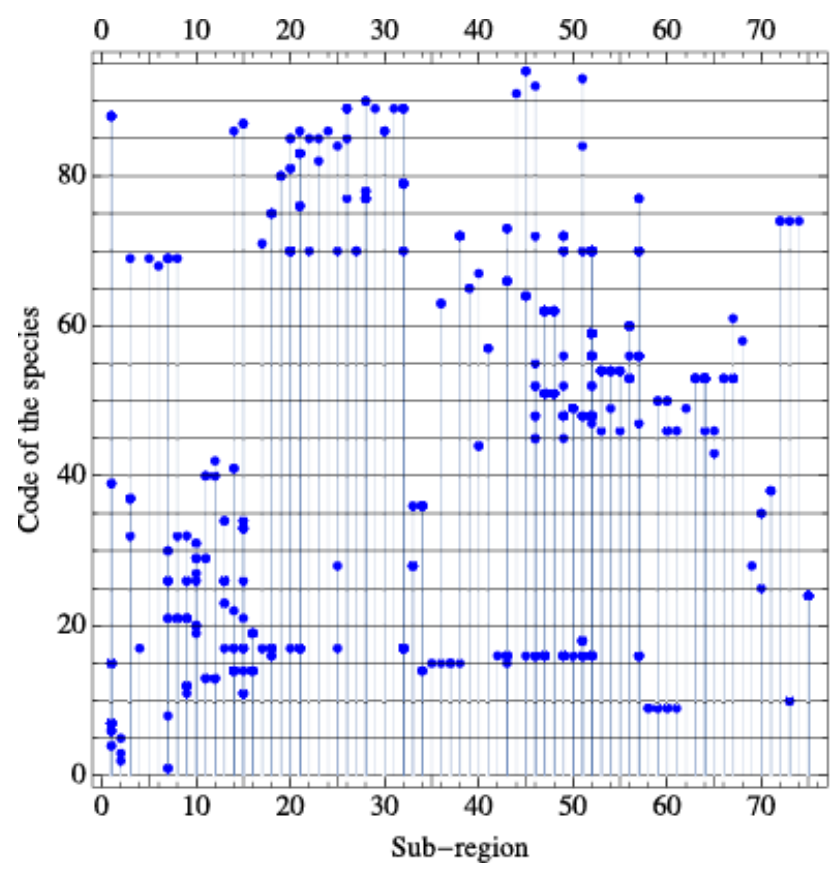

Figure 2. Distribution of the 94 species ( $y$-axis) in the 75 natural sub-regions ( $x$-axis) considered in this study. The number of points along a vertical line gives the number of species recorded in the selected sub-region, and the values of those points on the vertical axis identify the species by the codes assigned to them. Given a $y$-value, by following the horizontal line we identify the sub-regions where the species is recorded. For example, the species $(15 \Delta)$ Hypolobocera bouvieri angulata has been recorded for 6-subregions.

a combination of two contributions, species richness and abundance distribution.

The $B_{1}(S, r)$ biodiversity index used in this study allows the classification of the 75 sub-regions that we have considered, taking into account not only the number $S$ of species in the sub-region but also the relative abundances $p=\left\{p_{1}, p_{2}, \ldots, p_{S}\right\}$, where $p_{n}$ is the relation between the number of specimens of the $n$-th species and the total number of specimens of all species collected in the sub-region. As previously noted, due to the lack of standardization of the database used in this work, it may be only used as a proxy that resembles the relative abundance. In addition, although 94 freshwater crab species have been recorded for Colombia, number $S$ may change over time, either because new species are discovered or by the disappearance of some of them.

\section{ResUlts}

In relation to the history of the records and the description of the 94 species, we make a graph of the total number of species known $(S=94)$ in Colombia as a function of time (Fig. 3): one curve (circles) shows the year in which the first record of each species took place; the other curve (squares) shows the year in which the species was described in the literature. In both cases, these curves show that the initially rate of increase in the number of species observed (period 1897 to 1960) rises slightly, but increased significantly, after 1960. As more species are known, both curves should flatten and reach asymptotic values, which would indicate the approximate actual specific diversity of the Colombian pseudothelphusids.

The Fig. 3 corresponds to the sub-region San Juan river in the Western cordillera (15, CocSJ), where eight species coexist, and shows that the type material of the species was collected in some cases many years before its description. For example, Hypolobocera chocoensis (21) was described in 1980, whereas the type material was collected in 1910 and deposited at Natural History Museum, London (NHM). In the case of Hypolobocera rotundilobata (33), the holotype was collected in 1962, deposited at National Museum of Natural History, Smithsonian Institution (USNM), and the description took place in 1994.

As seen in Tab. 2, the sub-region with the largest number of records is the Eastern cordillera, Bogotá river (52, CorBog), followed by the Sierra Nevada of Santa Marta (1, Ssm), the Eastern cordillera, Negro river 1 (49, CorNeg1) and the Eastern cordillera, Suárez river (47, CorSua). This could indicate that the number of studies conducted in these sub-regions is larger than those in other localities. However, as noted in the Material and Methods Section, all subregions have been treated on an equal footing by using the average number of specimens captured in each sub-region.

In Tab. 2, the numerical codes in the database correspond to the species of the family Pseudothelphusidae. The non-endemic species are 


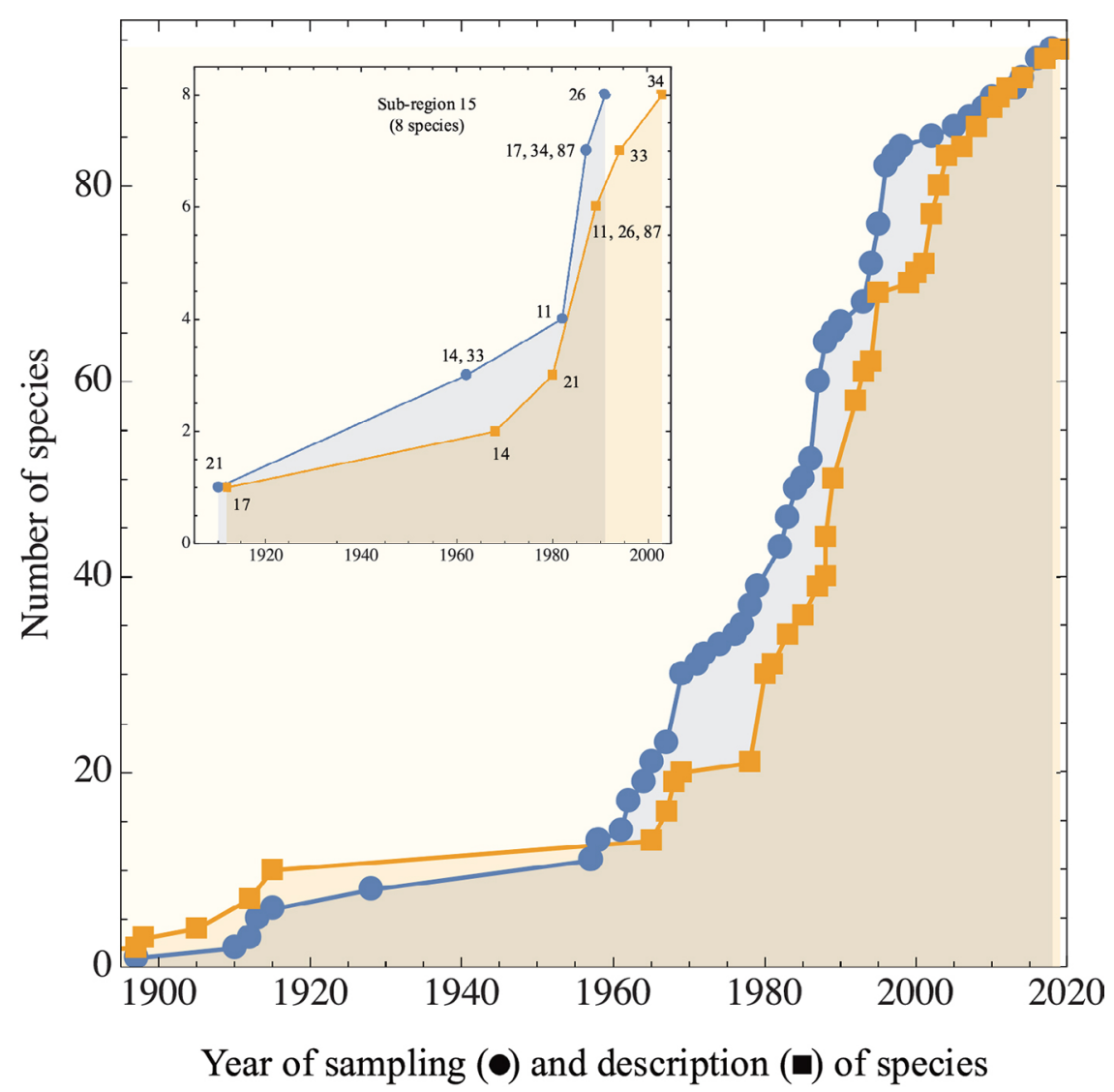

Figure 3. Accumulation curves showing the rates of increase in the number of new species of freshwater crabs in Colombia according to the year in which they were first recorded (circles and species code) and based on the year in which they were described in the literature (squares and species code). The inset refers to the sub-region San Juan river in the Western cordillera (15, CocSJ) in which 8 species coexist.

marked with symbol $\Delta$. The 6,263 specimens recorded in the database are distributed among the 94 species as indicated in the last column of Tab. 2. The numbers of specimens corresponding to endemic and nonendemic species are 6,093 and 170, respectively.

Species richness

If a sub-region contains a certain number of species $(\mathrm{N})$, we refer to it as a sub-region of species richness $\mathrm{N}$, expression for which we use the acronym "SR-N". The classification of the sub-regions is shown in Fig. 4.

SR-8 sub-regions - Among the 75 natural subregions considered, San Juan river in the Western cordillera (15, CocSJ) is the sub-region with the highest number of species, and it is represented in the database by 305 specimens belonging to eight species. Note that these species are not exclusive to this sub-region because there are 830 records in the database, which are distributed as shown in Tab. 2 . Hypolobocera rotundilobata (33) and Strengeriana risaraldensis (87) are endemic to the San Juan river (15, CocSJ), while the other seven species (Sp) are also present in other sub-regions, namely: Hypolobocera alata (11), (9, CbioSJ), (15, CocSJ); Hypolobocera beieri (14), (14, CocCau), (15, CocSJ), (16, CocPac) and (34, CcePat); Hypolobocera bouvieri monticola (17), (4, CaribeCau), (13, CocAt ), (14, CocCau), (15, CocSJ), (17, CceNare ), (18, CceMed), (20, CceMiel), (21, CceCau), (25, СсеMa), (32, CceAmo); $H$. chocoensis (21), (7, CbioAt), (8, CbioBau), (9, CbioSJ); Hypolobocera lloroensis (26), (7, CbioAt), (9, CbioSJ), (10, CbioPac), and H. velezi (34), (13, CocAt), (15, CocSJ). Based on the aforementioned list and on the map (Fig. 1), we established that the subspecies $H$. bouvieri monticola (17) occurred in ten sub-regions, having then the widest geographic distribution in Colombia (Fig. 2). 


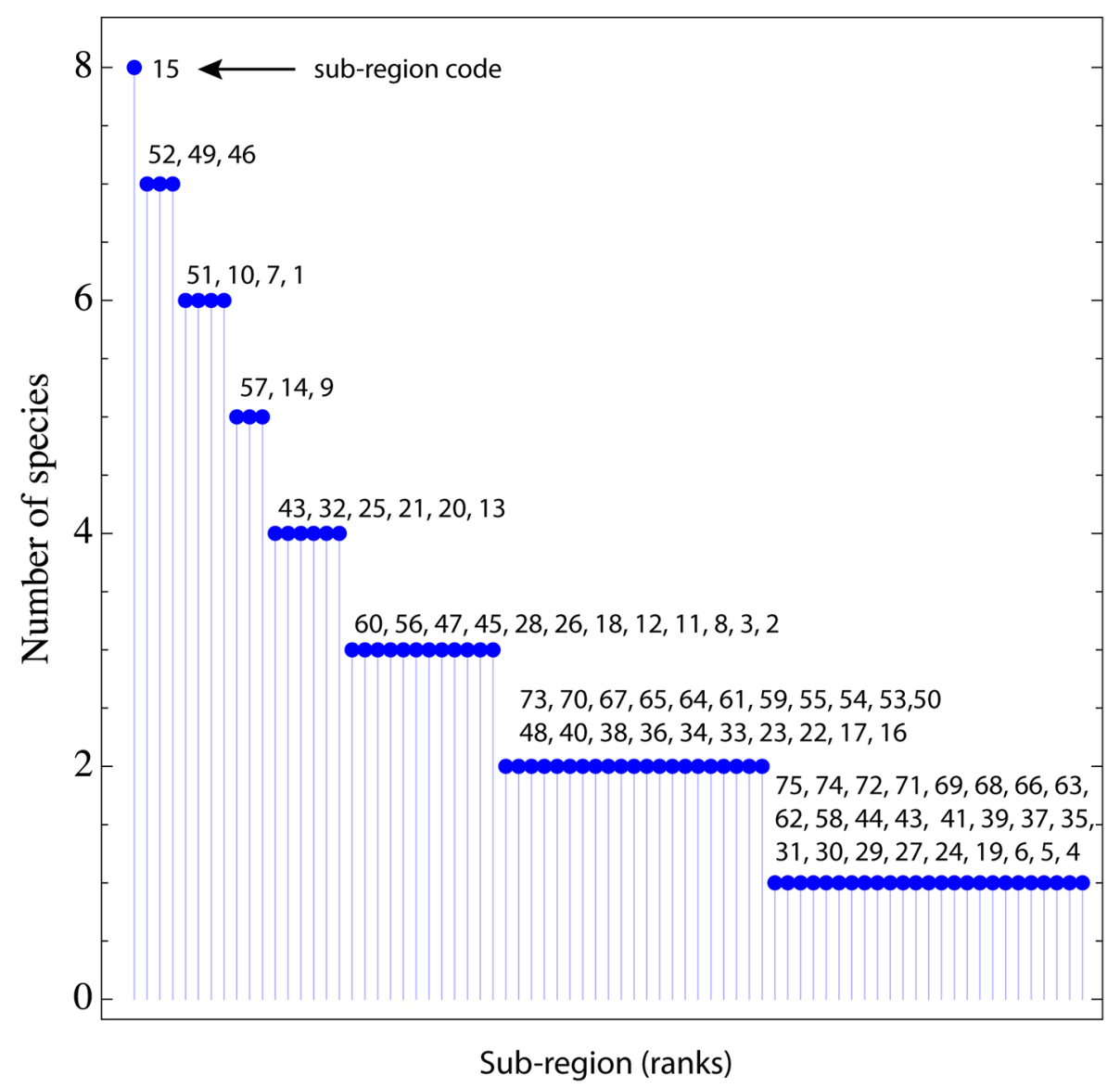

Figure 4. Natural sub-regions ranked in terms of species richness.

SR-7 sub-regions - Seven species were recorded in the three sub-regions: Minero river (46, CorMin), Negro river 1 (49, CorNeg1) and Bogotá river (52, CorBog), all located in the Eastern cordillera, and the rivers are tributaries of the Magdalena river. The basic information on these sub-regions is shown in Tab. 2.

Neostrengeria lemaitrei (55) and N.fernandezi (92) are restricted to the sub-region (46, CorMin). The three SR-7 sub-regions have the following in common: H. bouvieribouvieri (16), Neostrengeria botti (48) and N. gilberti (52); sub-regions (46, CorMin) and (49, CorNeg1) share the species Neostrengeria aspera (45) and Phallangothelphusa magdalenensis (72), while the (49, CorNeg1) and (52, CorBog) sub-regions share the species Neostrengeria lindigiana (56) and Phallangothelphusa dispar (70). Neostrengeria gilberti (52) is an endemic species restricted to the SR-7 subregions, and Neostrengeria macropa (59) is endemic to (52, CorBog).

SR-6 sub-regions - There are four sub-regions involved, namely: Sierra Nevada de Santa Marta (1,
Ssm), Chocó Biogeographic, Atrato river (7, CbioAt), Chocó Biogeographic, Pacific (10, CbioPac), and Eastern cordillera, Magdalena river (51, CorMa).

The Sierra Nevada de Santa Marta $(1, \mathrm{Ssm})$ is an isolated mountain range located about 42 kilometers from the Caribbean coast; it is separated from the Andean Chain, and reaches an elevation of 5,700 $\mathrm{m}$. This mountain range has a large number of ecoregions associated with its altitude. With the exception of Hypolobocera bouvieri angulata (15), all the species present in the Sierra Nevada de Santa Marta are endemic to that region, namely: Chaceus davidi (4), Chaceus nasutus (6), Chaceus pearsei (7), Martiana clausa (39) and Strengeriana taironae (88).

Although the Chocó Biogeographic, Atrato river (7, CbioAt) and the Chocó Biogeographic, Pacific (10, CbioPac) sub-regions are located in the Pacific region, they do not share any species. Based on the current records in $(10, \mathrm{CbioPac})$ there are three endemic species: Hypolobocera murindensis (30), Hypolobocera malaguena (27), and Hypolobocera 
mutisi (31), whereas in (7, CbioAt) there are only two endemic species: Colombiathelphusa culmarcuata (1) and $H$. murindensis (30).

The Magdalena river is historically the most important in Colombia (Galvis and Mojica, 2007), beginning its course in San Agustin, Huila Department, to the southwest of Colombia in the Andes mountain range, along the central axis of the Colombian massif. The river crosses the country through the west from south to north, in a distance about $1,540 \mathrm{~km}$ between the Eastern and Central cordilleras of the Colombian Andes, forming a valley (extension 200,000 $\mathrm{km}^{2}$ ) that arrives at the coast of the Caribbean Sea. In this study, the sub-regions (51, CorMa) and (38, MmeMa) are associated with the upper and middle parts of the Magdalena river. The species Neostrengeria lassoi (93) and the subspecies Hypolobocera bouvieri estenolobata (18) are endemics to the sub-region (51, CorMa).

SR-5 sub-regions - There are three sub-regions involved, namely: Eastern cordillera, Sumapaz river (57, CorSum), Western cordillera, Cauca river (14, CocCau) and Chocó Biogeographic, San Juan river (9, CbioSJ). The first two sub-regions are in the Andean region, whereas the last one is in the Pacific region. The Sumapaz and Cauca rivers are tributaries of the Magdalena river, whereas the San Juan river flows into the Pacific Ocean; the Cauca river is considered the second most important in Colombia. These subregions do not share any species with each other, but their species are present in other sub-regions, except Hypolobocera dentata (22) in (14, CorCau); Hypolobocera andagoensis (12) and H. chocoensis (21) in (9, CbioSJ), which are exclusive to these regions.

SR-4 sub-regions - In this case, we find six sub-regions: on one side, the Eastern cordillera, Lebrija river (43, CorLebr) and Western cordillera, Atrato river (13, CocAtr) and on the other side, the Amoyá river (32, CceAmo), Magdalena river (25, $\mathrm{CceMa}), \mathrm{Cauca}$ river (21, CceCau) and Miel river (20, CceMiel) all located in the Central cordillera. Regarding the SR-4 sub-regions it is important to mention: (i) Hypolobocera bouvieri monticola (17) is not recorded for (43, CorLebr), but it is shared by the other five sub-regions. (ii) Phallangothelphusa dispar (70) occurs in sub-regions (32, CceAmo), (25, CceMa) and (20, CceMiel). (iii) Neostrengeria tonensis
(66) and Phallangothelphusa martensis (73) are only recorded for (43, CorLebr); similarly, Strengeriana fuhrmanni (83) and Strengeriana bolivarensis (76) are restricted to (43, CorLebr). (iv) The following species are restricted to one sub-region, namely: Strengeriana chaparralensis (79) to (32, CceAmo), Strengeriana florenciae (81) to (20, CceMiel), and Hypolobocera emberarum (23) to (13, CocAt).

SR-3 sub-regions - In this case there are 12 sub-regions: Negro river 2 (56, CorNeg2), Suárez river (47, CorSua) and Opón river (45, CorOpon) belong to the Eastern cordillera, whereas Azufrado river (28, CceAzu), Coello river (26, CceCoe) and Medellín river $(18$, CceMed) are located in the Central cordillera. The sub-regions Telembí river (12, CbioTel), Patía river (11, CbioPat) and Baudó river (8, CbioBau) are situated in the Biogeographic Chocó, whereas the Sinú river (3,CaribbeanSi) and Cesar river (2, CaribbeanCes) correspond to the Caribbean region, and Cusiana river (60, OriCus) is located in the Orinoquian region.

Among the SR-3 subregions we found a high degree of endemism, namely: (i) Chaceus cesarensis (2), Ch. curumanensis (3) and Chaceus ibiricensis (5) are endemic to (2, CaribeCes). (ii) Strengeriana casallasi (78) and Strengeriana villaensis (90) are restricted to (28, CceAzu). (iii) Neostrengeria sketi (64) and Phallangothelphusa tangerina (94) are endemic to (45, CorOpon). (iv) Lindacatalina sinuensis (37), Moritschus narinnensis (42) and Strengeriana antioquensis (75) are endemic only to (3, CaribeSi), (12, CbioTel) and (18, CceMed), respectively. (v) The sub-regions (47, CorSua), (45, CorOpon), (18, CceMed) have in common $H$. bouvieri bouvieri (16), whereas $(28, \mathrm{CceAzu})$ and $(26, \mathrm{CceCoe})$ share the species Strengeriana cajaensis (77).

SR-2 sub-regions - In the 21 sub-regionsweidentify the following particularities: (i) we found species with restricted distribution, namely: Hypolobocera kamsarum (25) and Lindacatalina latipenis (35) to (70, CorCa), Neostrengeria appressa (44) and Orthothelphusa holthuisi (67) to (40, CorTach), Fredius granulatus (10) to (73, AmazoApa), Neostrengeria natashae (61) to (67, OriAca), Neostrengeria alexae (43) to (65, OriHum), Neostrengeria perijaensis (63) to (36, CorTuc), Strengeriana foresti (82) to (23, CceGuar), and Phallangothelphusa juansei (71) to 
(17, CceNare). (ii) At least two or more subregions share one species, specifically: (65, OriHum), (64, CorGuat), (61, OriTua), (55, CorGua) and (53, CorBat), Neostrengeria bataensis (46); (55, CorGua), (54, CorGar) and (53, CorBat), Neostrengeria lasallei (54); (67, OriAca) and (64, CorGuat), Neostrengeria guenteri (53); (54, CorGar) and (50, CorChi), Neostrengeria boyacensis (49); (61, OriTua) and (59, OriCra), Eudaniela casanarensis (9); (38, MmeMa) and (36, CorTuc), H. bouvieri angulata (15); (34, CcePat) and (16, CocPac), H. beieri (14); (34, CcePat) and (33, CcePaez), Lindacatalina orientalis (36); (23, CceGuar) and (22, CceGual), Strengeriana maniformis (85).

\section{Species diversity index}

The biodiversity index $B_{1}(S, r)$ was calculated for the 75 sub-regions considered in this study, where $S \rightarrow S_{n}$ and $r \rightarrow r_{n}$ are the number of species and the radius associated with the $n$-th sub-region, and the index $n$ runs on all sub-regions $(n=1,2, \ldots, 75)$. The values of $\alpha_{1}(S)$ required for the calculation of the index $B_{1}(S, r)$ are given by $0.63662,0.75,0.848826$, 0.9375, 1.01859, 1.09375, 1.1641, 1.23047, 3.89865, which correspond to the number of species $S=1,2$, $3,4,5,6,7,8$, and 94 , respectively.

In Fig. 5, we make use of the biodiversity plane in which $x$-axis is the radius $r=\sqrt{D}$ and $y$-axis is the biodiversity index $B_{1}(S, r)$. The solid curves represent the function $\alpha(S) / r$, where the value of the constant $\alpha(S)$ is determined by the number $S$ of species (iso-species curve or $S$-curve). In this way, points of the biodiversity index for two or more sub-regions that have the same species richness $S$, are located exactly on the same $S$-curve. While the representative points remain on the same $S$-curve, their positions on the $r$-axis may differ if the species have different abundance distributions. In Fig. 5, the lowest point on the right hand-side represents the 25 sub-regions of species diversity one (SR-1).

Based on the values of the $B_{1}(S, r)$ index (Fig. 5) and the ranges proposed in Tab. 3 , the sub-regions were classified according to the degree of diversity.

Some additional comments are relevant with respect to Tab. 3: (i) The six High biodiversity $(\mathrm{Hb})$ sub-regions (52, CorBog), (49, CorNeg1) and (7, CbioAt) belong to the SR-7 sub-regions while (10, CbioPac), (51, CorMa) and (46, CorMin) are of the
SR-6 sub-regions (Fig. 4). In Fig. 5 they are represented in the high biodiversity area by three points on the curve $S=7$ and three points on the curve $S=6$, respectively. (ii) The five Medium biodiversity (Meb) sub-regions (15, CocSJ), (1, Ssm), (57, CorSum), (20, CceMiel) and (13, CocAt), are respectively of SR-8, SR-6, SR-5 sub-regions, whereas the last two are SR-4. (iii) Moderate biodiversity (Mob) sub-regions 9 and 14 are characterized by the presence of 5 species in each of them, unlike the other sub-regions belonging to this class that only recorded 4,3 or 2 species. (iv) Similarly, sub-regions with low biodiversity (Lob) record two species, except the sub-region (8, CbioBau) that presents 3 species. (v) The 25 Scarce biodiversity (Scb) SR-1 sub-regions are characterized by having a biodiversity index with a common value of approximately 0.637 .

Finally, the following results on freshwater crabs of the family Pseudothelphusidae in Colombia are also relevant: (i) in relation to the 75 natural subregions used in this study, there are 25 sub-regions in which the pseudothelphusid diversity is low, because a single species occurs in each one, and for five of these species only the holotype is known: H. malaguena

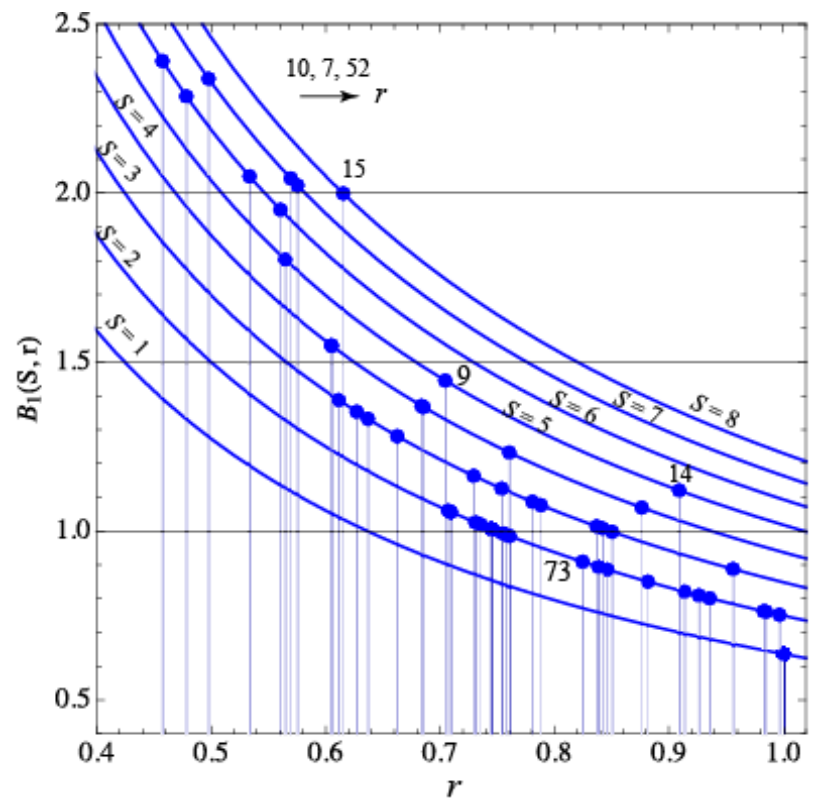

Figure 5. Comparison of natural sub-regions defined in Table 1 in terms of the biodiversity index $B_{1}(S, r)$ as a function of radius $r=\sqrt{D}$, where $D$ is the Simpson index. The numbers in the vertical lines identify some sub-regions $(15,9,14,73)$; the representative points of them are located on iso-species curves ( $S$-curves), according to the $S$ number of species present in the sub-region. 
Table 3. Classification of the natural sub-regions from Colombia, on a scale of levels, according to the value of the $B_{1}(S, r)$ biodiversity index. The sub-regions are identified by their codes and are listed in order of decreasing biodiversity.

\begin{tabular}{cccc}
\hline Degree & Descriptor & Range of $\boldsymbol{B}_{1}(\boldsymbol{S}, \boldsymbol{r})$ & Sub-regions \\
\hline $\mathrm{Hb}$ & High biodiversity & $2<B_{1}(S, r) \leq 2.5$ & $10,52,7,51,49,46$ \\
$\mathrm{Meb}$ & Median biodiversity & $1.5<B_{1}(S, r) \leq 2$ & $15,1,57,20,13$ \\
$\mathrm{Mob}$ & Moderate biodiversity & $1<B_{1}(S, r) \leq 1.5$ & $9,12,43,32,60,45,11,21,56,47,14,28,18,25,23$, \\
$\mathrm{Lob}$ & Low biodiversity & $0.75<B_{1}(S, r) \leq 1$ & $36,55,70,26,3,54,33,22,17$ \\
$\mathrm{Scb}$ & Scarce biodiversity & $0<B_{1}(S, r) \leq 0.75$ & $8,16,65,34,73,40,2,48,50,61,67,59,53,38,64$ \\
\hline
\end{tabular}

(27), Neostrengeria macarenae (58), Potamocarcinus colombiensis (68), S. foresti (82) and Neostrengeria libradensis (91). (ii) There are also 15 sub-regions with low biodiversity: in 14 sub-regions there are only two species, but they are represented in the database by a large number of specimens.

\section{Discussion}

In this study concerning the species of the family Pseudothelphusidae in Colombia, we began by asking about the possibility of obtaining useful information from the natural history collections existing in museums and research institutions.

A few studies using databases of natural history collections to assess the regional diversity of freshwater crabs have been carried in Mexico on the basis of data maintained by the Colección Nacional de Crustáceos (CNCR), Instituto de Biología, Universidad Nacional Autónoma de México. Álvarez et al. (1999) presented a list of 335 species of decapod crustaceans from Veracruz (marine, estuary and freshwater), and found that freshwater decapods represent $28 \%$ of the total number of species registered for Mexico, of which 11 are pseudothelphusid species. Álvarez et al. (2005) provide an annotated list of crustaceans in the continental water bodies of the Mexican state of Tabasco and found that out of 72 recorded species 25 correspond to freshwater decapods and only four belong to the family Pseudothelphusidae. Using information from the CNCR and the Museo de Zoología, Universidad de Ciencias y Artes de Chiapas in Tuxtla Gutiérrez, Chiapas, Álvarez et al. (2011) presented a taxonomic treatment of the 80 species of freshwater and land crustaceans that are distributed in Chiapas. In this Mexican state there are three of the five tribes that constitute the family Pseudothelphusidae: Hypolobocerini (2 spp.), Potamocarcinini (11 spp.), and Pseudothelphusini (1 sp.). Álvarez et al. (2014), through an exhaustive compilation of the decapod crustacean records from Mexico, built a species list for the continental, Caribbean, Gulf of Mexico and Pacific regions that includes a total of 1,775 species. They also considered that, based on projections proposed by other authors, it is estimated that only half of the species of decapods that occur in Mexico were then known.

In the present study we find that: (i) Four tribes have been described for Colombia; Hypolobocerini (60 spp.) is distributed in the five natural regions of Continental Colombia and Gorgona Island; Kingsleyini (4 spp.) occurs in the Andean, Orinoquian and Amazonian regions; Potamocarcinini (2 spp.) has been recorded from Caribbean and Pacific regions, and Strengerianini (28 spp.) has been found in the Caribbean and Andean regions. (ii) The distribution of these tribes is not homogeneous and, within the set of 75 sub-regions, they are represented in 58, 9, 4 and 28 sub-regions, respectively, a fact that is explained by the biogeographical and ecological conditions of the country, and the specific environmental conditions that species require to survive. (iii) In the Western cordillera, San Juan river (15, CocSJ), the sub-region in which the largest number of species was recorded, there are seven Hypolobocerini species and only one Strengerianini. (iv) In sub-regions Cravo Sur river, (59, OriCra), Cusina river (60, OriCus) and Túa river (61, OriTua), located in the Orinoquian natural 
region, the tribes Kingsleyini and Hypolobocerini coexist, whereas for the sub-regions Guaviare river (72, AmazoGua), Apaporis river (73, AmazoApa) and Caquetá river (74, AmazoCa) of the Amazonian natural region, only species of Kingsleyini are recorded. (v) In the Pacific natural region, the tribes Potamocarcinini and Hypolobocerini coexist in three sub-regions, Sinú river (3, CaribeSi) of the Caribbean natural region, Atrato river (7, CbioAt) and Baudó river (8, CbioBau) of the Chocó Biogeographic, whereas in subregions León river (5, CbioLeo) and Juradó river (6, CbioJu) of the Chocó Biogeographic, the known records are only for Potamocarcinini. (vi) Comparing the previous facts with the information corresponding to Chiapas (Álvarez et al., 2011), it is evident that in the Mexican region there are very diverse biogeographic and ecological conditions that allow the coexistence of three tribes, inasmuch as its area is only $3.8 \%$ of Mexico or $6.5 \%$ of Colombia (continental areas).

Based on the faunistic and taxonomic studies by Magalhães (1990; 2005; 2009; 2017), Magalhães and Pereira (2007), Magalhães and Türkay (2010), Pedraza et al. (2016), Pedraza and Tavares (2015), Pereira et al. (2009; 2010), Suárez (2015), Magalhães et al. (2014; 2018), and Zanetti et al. (2018), one can count the number of pseudothelphusid species occurring in the Orinoco and Brazilian Amazon basins. Cross-referencing our data with the number of species obtained from those studies, we can notice that (i) the Negro river, the largest northern tributary of the Amazon river in Brazil, which also includes the Branco river drainage, has thirteen species distributed in four genera: Fredius (8 spp.), Kingsleya (2 spp.), Microthelphusa (2 spp.), and Prionothelphusa (1 sp.); (ii) twenty-one species (with two subspecies) in eight genera are recorded from the Orinoco basin: Microthelphusa (7 spp.), Fredius (7 spp. + 2 spp.), Eudaniela, Kingsleya, Oedothelphusa, Orthothelphusa, Prionothelphusa, and Rodriguezus (1 sp. each); (iii) five species are known from the Xingu river: four of Kingsleya and one of "Microthelphusa"; (iv) eight species of four genera (Kingsleya, 3 spp.; Fredius, 2 spp.; Melothelphusa, 2 spp.; Brasiliothelphusa, 1 sp.), additionally occur in other tributaries of the Amazon river (Magalhães, 1986; 2005; 2009; 2017; Magalhães and Türkay, 2010; Magalhães et al., 2014;
Pedraza and Tavares, 2015; Pedraza et al., 2016). On the other hand, in the sub-regions (72) AmazoGua, (73) AmazoApa and (74) AmazoCa considered in this study for the Colombian Amazon region, there are only two species recorded: (a) Prionothelphusa eliasi occurs in Colombia, but also in Venezuela and Brazil, in tributaries of the Amazon and Orinoco basins, and (b) Fredius granulatus is only recorded for Colombia, in Mirití-Paraná river, which is a tributary of the Amazon river. Regarding the Colombian Orinoquian region, eight sub-regions were established, which are characterized by being tributaries of the Meta river and which flows into the Orinoco river: (58) OriPau, (59) OriCra, (60) OriCus, (61) OriTua, (65) OriHum, (66) OriAri, (67) OriAca and (68) OriGue. Seven species are recorded, six of the genus Neostrengeria and one of Eudaniela. It is notable that E. casanarensis has a distribution that extends to 4 sub-regions (58 to 61) while $N$. bataensis occurs in three sub-regions $(60,61,65)$. Recently an unidentified female of the genus Kingsleya was collected in Puerto Carreño, Vichada, on the Orinoco river, which is important insofar as it constitutes the first record of this genus for Colombia. In short, 21 (with 2 subspecies) and 8 pseudothelphusid species have been recorded from the Venezuelan and Colombian portions of the Orinoco basin, respectively. The Amazon basin in Brazil has 21 recorded species, but only two in the Colombian Amazon region. According to this data, the basins of the three countries differ in species richness, which can be explained by insufficient sampling/uneven collecting efforts or by diverse ecological conditions/ geological history, or by both.

In the light of the Colombian natural sub-regions considered in this work, the Andean region (51 subregions), ranks first regarding the pseudothelphusid species richness, with 53 species $(56.4 \%)$ recorded out of the 94 species identified for Continental Colombia and Gorgona Island, seconded by the Pacific region (8 sub-regions) with 19 species (20.2\%). These regions are followed by the Caribbean region (4 subregions) with 13 species (13.8\%), the Orinoquian region (8 subregions) with 7 species (7.5\%), and the Amazon region (4 sub-regions) with 2 species (2.1\%). Mountain regions are unusually biodiverse, with numerous aggregations of narrow-ranged species that form centers of endemism, especially in the tropics (Rahbek 
et al., 2019). This is the case for the Colombian Andean region, that, with its Western, Central and Eastern mountain ranges, forms a topographically complex natural region, with diverse and distinct mountainous environments, which could account for the highest species richness of the Andean region verified in the present study. This fact is also consistent with the statement by Rodriguez (1982) that the pseudothelphusids are predominantly a montane group, with many of their species having narrowrange distributions and that could be explained by the high degree of speciation resulting from geographical isolation in several sub-basins in the Andean mountainous regions.

The present analysis covers a wide period (1853 to 2019) of collecting samples of the Colombian pseudothelphusids. The natural environmental conditions were not stable during that period due to natural events or anthropogenic activities such as deforestation, mining, agriculture, pollution, and so on. Therefore, the obtained results do not describe the current state of pseudothelphusid populations for Colombia. As aforementioned, the average number of specimens captured in each sub-region was used for the analyses. Notwithstanding, for future studies it is pertinent to reduce bias of new information by collecting data using a more appropriate methodology that would permit rapid surveys to evaluate biotic complementarity as well as promote long-term ecological studies such as the RAPELD method (Magnusson et al., 2005).

The curves of species accumulation depicted in Fig. 3 clearly shows that they have not reached their respective asymptotes, which suggests that more comprehensive biological surveys are needed as there may be yet undiscovered species of freshwater crabs in Colombia.

Regarding the 75 selected sub-regions, one can observe that: (i) Twenty five of the 94 species have very restricted distributions, being found in only one of the sub-regions. This is the case, for instance, of Ch. davidi (4), which only occurs in (1, Ssm). (ii) There are species widely distributed as they share several sub-regions, for example: $H$. bouvierimonticola (17) is recorded in ten sub-regions: (4, CaribeCau), (13, CocAt ), (14, CocCau), (15, CocSJ), (17, CceNare ), (18, CceMed), (20, CceMiel), (21, CceCau), (25, CceMa), (32,
CceAmo), having the widest geographic distribution in Colombia; and N. bataensis (46) is presented in 5 sub-regions: (65, OriHum), (64, CorGuat), (61, OriTua ), (55, CorGua) and (53, CorBat). (iii) Some species converge in the same sub-region, as occurs with 8 species in (15, CocSJ). Thus, regarding species with very restricted distributions, the selection of the 75 sub-regions has been an appropriate instrument, since it implies the importance of conducting further studies on the particular environments in which these species inhabit.

The 94 species of the family Pseudothelphusidae are represented in the database with a varied number of specimens. This is explained by the environmental conditions of their habitat, and by the number and intensity of the sampling in the distributional range of the species. This methodological bias indicates that sampling efforts should be increased for those species represented in the database by a low number of specimens. As special cases, two species described by Rodríguez (1980), N. libradensis (91) and S. foresti (83), are based on single specimens and, despite explorations in localities near the type locality, have not had any additional material collected. A similar situation occurs with $N$. macarenae (58), collected in the sub-region Orinoquian, Güejar river (68, OriGue), for which the only specimen known is the holotype. Despite exploration performed in the type locality, it has not been possible to find additional specimens.

According to Cumberlidge (2016), there are approximately 6,800 known species of brachyuran crabs, about 1,400 (20.6\%) have been reported from freshwater habitats, and 271 of them are known species of freshwater crabs of the Neotropical family Pseudothelphusidae. Thus, of 94 existing species in Colombia, 86 are endemic and they correspond to $31.7 \%$ of the total pseudothelphusid species. Therefore, the conservation of their environment is a priority for the preservation of the species diversity of the family, responsibility that should be shared by the scientific community, Colombian environmental governmental and non-governmental organizations, and the people as a whole.

It should be noted that the index of diversity of species $B_{1}(S, r)$ allows for a quantitative classification of the sub-regions, since it depends not only on the number of species but also on their 
abundance distributions. Again, appropriate sampling and additional studies are required to improve the reliability in the classification, based on the index and a scale of values such as the one in Tab. 3 .

The high diversity of freshwater crab species in Colombia may be explained by the heterogeneity of existing natural environments in the continental country and on Gorgona Island, which led to the 75 natural sub-regions considered in this study. The analysis carried out in each one of the sub-regions in relation to their number of species constitutes a baseline for subsequent investigations that tend to consolidate the information on the actual number of species. The results here obtained are useful for designing new research proposals on conservation and environmental impact studies concerning the family Pseudothelphusidae, in particular for sub-regions in which only one species is recorded.

\section{AcKNOWLedgmenTS}

The authors would like to gratefully acknowledge Dr. Célio Magalhães, Dr. Christopher Tudge, and the anonymous referees for valuable input and constructive comments on the manuscript. Our special thanks to Gian Piero Fiorentino for a grammatical revision of the text and Rubén Albarracín for the elaboration of the map.

\section{RefERENCES}

Alvarado, M. 2011. Regiones Naturales de Colombia. Available at https://www.calameo.com/read/0012450029a549c75c5d3. Accessed on 4 June 2020.

Álvarez, F.; Villalobos, J.L. and Robles, R. 2005. Crustáceos. p. 177-194. In: J. Bueno; F. Álvarez and S. Santiago (eds), Biodiversidad del Estado de Tabasco. México, D.F., Instituto de Biología, UNAM-CONABIO.

Álvarez, F.; Villalobos, J.L.; Elías-Gutiérrez, M. and Rivera, G. 2011. Crustáceos dulceacuícolas y terrestres de Chiapas. p. 209-297. In: F. Álvarez (coord.), Chiapas. Estudios sobre su diversidad biológica. México, Universidad Nacional Autónoma de México.

Álvarez, F.; Villalobos, J.L.; Rojas, Y. and Robles, R. 1999. Lista y comentarios sobre los crustáceos decápodos de Veracruz, México. Anales del Instituto de Biología Universidad Nacional Autónoma de México, Serie Zoología, 70: 1-27.

Álvarez, F.; Villalobos, J.L.; Hendrickx, M.E.; Escobar-Briones, E.; Rodríguez-Almaraz, G. and Campos, E. 2014. Biodiversidad de crustáceos decápodos (Crustacea: Decapoda) en México. Revista Mexicana de Biodiversidad, Supl. 85: 208-219.

Bott, R. 1967. Fluss-krabben aus dem westlichen Südamerika. Senckenbergiana biologica, 48: 365-372.

Campos, D. and Isaza, J.F. 2009. A geometrical index for measuring species diversity. Ecological Indicators, 9: 651-658.

Campos, D. and Campos, M.R. 2012. Underlying thermodynamic relations of a species diversity index: Freshwater crabs from Colombia. Ecological Indicators, 15: 198-207.

Campos, M.R. 1989. Nuevas especies de cangrejos de agua dulce del género Hypolobocera (Crustacea: Decapoda: Pseudothelphusidae) para Colombia. Trianea, 3: 143-147.

Campos, M.R. 1992. New species of fresh-water crabs of the genus Neostrengeria Pretzmann, 1965 (Crustacea: Decapoda: Pseudothelphusidae) from Colombia. Proceedings of the Biological Society of Washington, 105: 540-554.

Campos, M.R. 1995. A new species of fresh-water crab of the genus Strengeriana from Colombia (Crustacea: Decapoda: Pseudothelphusidae) from Colombia. Proceedings of the Biological Society of Washington, 108: 98-101.

Campos, M.R. 1998. A new species of freshwater crab of the genus Phallangothelphusa Pretzmann, 1965 from Colombia (Crustacea: Decapoda: Pseudothelphusidae). Proceedings of the Biological Society of Washington, 111: 92-96.

Campos, M.R. 1999. A new species of freshwater crab of the genus Strengeriana Pretzmann, 1971, from Colombia (Crustacea: Decapoda: Pseudothelphusidae). Proceedings of the Biological Society of Washington, 112: 405-409.

Campos, M.R. 2000. Neostrengeria binderi, a new species of pseudothelphusid crab from the eastern Andes of Colombia (Crustacea: Decapoda: Brachyura). Proceedings of the Biological Society of Washington, 113: 401-405.

Campos, M.R. 2001. A new genus and species of freshwater crab from Colombia (Crustacea: Decapoda: Pseudothelphusidae). Proceedings of the Biological Society of Washington, 114: 938943.

Campos, M.R. 2003. A new species of freshwater crab of the genus Potamocarcinus H. Milne Edwards, 1853 (Crustacea: Decapoda: Brachyura: Pseudothelphusidae), from Colombia. Academia Colombiana de Ciencias Exactas Físicas y Naturales, 27: 283-286.

Campos, M.R. 2004. Neostrengeria lemaitrei, a new species of freshwater crab from Colombia. Proceedings of the Biological Society of Washington, 117: 363-367.

Campos, M.R. 2010a. A new species of freshwater crab of the genus Phallangothelphusa Pretzmann, 1965, from Colombia (Crustacea: Decapoda: Pseudothelphusidae), with a key to the species of the genus. Proceedings of the Biological Society of Washington, 123: 27-31.

Campos, M.R. 2010b. Anew species of freshwater crab of the genus Neostrengeria Pretzmann, 1965, from Colombia (Crustacea: Decapoda: Pseudothelphusidae). Zootaxa, 2482: 64-68.

Campos, M.R. 2011. A new species of freshwater crab of the genus Neostrengeria Pretzmann, 1965, from Meta Department, Colombia (Crustacea: Decapoda: Pseudothelphusidae). Caldasia, 33: 659-664. 
Campos, M.R. 2014. Crustáceos decápodos de agua dulce de Colombia. Bogotá, Instituto de Ciencias Naturales, 692p. (Serie José Jerónimo Triana, 27).

Campos, M.R. 2017. Two new species of freshwater, cavedwelling crabs of the genus Neostrengeria Pretzmann, 1965, from Colombia (Crustacea: Decapoda: Pseudothelphusidae). Zootaxa, 4247: 157-164.

Campos, M.R. and Campos, D. 2017. Species diversity of freshwater Decapod Crustaceans (crabs and shrimps) from Colombia. Crustaceana, 90: 883-908.

Campos, M.R. and Lemaitre, R. 1998. A new freshwater crab of the genus Neostrengeria Pretzmann, 1965, from Colombia (Crustacea: Decapoda: Brachyura: Pseudothelphusidae), with a key to the species of the genus. Proceedings of the Biological Society of Washington, 111: 899-907.

Campos, M.R. and Magalhães C. 2014. Colombiathelphusa, a new genus of freshwater crab from Colombia, and the first location record of Eidocamptophallus chacei (Pretzmann, 1967) (Crustacea: Decapoda: Pseudothelphusidae).Zootaxa, 3860: 571-579.

Campos, M.R. and Pedraza, M. 2006. A new species of freshwater crab of the genus Strengeriana Pretzmann, 1971, from Colombia (Crustacea: Decapoda: Pseudothelphusidae), with an updated key to the species of the genus. Proceedings of the Biological Society of Washington, 119: 557-562.

Campos, M.R. and Pedraza, M. 2008. Two new species of freshwater crab of the genus Neostrengeria Pretzmann, 1965, from Colombia (Crustacea: Decapoda: Pseudothelphusidae), with an updated key of the species of the genus. Caldasia, 30: 457-468.

Campos, M.R. and Rodríguez, G. 1984. New species of freshwater crabs (Crustacea: Decapoda: Pseudothelphusidae) from Colombia. Proceedings of the Biological Society of Washington, 97: 540-545.

Campos, M.R. and Rodríguez, G. 1985. A new species of Neostrengeria (Crustacea: Decapoda: Pseudothelphusidae) with notes on geographical distribution of the genus. Proceedings of the Biological Society of Washington, 98: 718-727.

Campos, M.R. and Rodríguez, G. 1988. Notes on the freshwater crabs of the genus Moritschus Pretzmann, 1965 (Crustacea: Decapoda: Pseudothelphusidae) with description of $M$. narinnensis from Southern Colombia. Proceedings of the Biological Society of Washington, 101: 640-643.

Campos, M.R. and Rodríguez, G. 1993. Three new species of Strengeriana from Colombia (Crustacea: Decapoda: Pseudothelphusidae). Proceedings of the Biological Society of Washington, 106: 508-513.

Campos, M.R. and Rodríguez, G. 1995. Two new species of freshwater crabs of the genus Hypolobocera from Colombia (Crustacea: Decapoda: Pseudothelphusidae). Proceedings of the Biological Society of Washington, 108: 649-655.

Campos, M.R. and Valencia D.M. 2004. Two new species of freshwater crabs of the genus Chaceus Pretzmann, 1965 from the Serranía de Perijá of Colombia (Crustacea: Decapoda: Pseudothelphusidae). Proceedings of the Biological Society of Washington, 117: 35-41.

Campos, M.R.; Lasso, C.A. and Arias, M. 2019. A new species of freshwater crab of the genus Phallangothelphusa Pretzmann, 1965, from the foothills of the Serranía Yariguíes of Colombia
(Crustacea: Decapoda: Pseudothelphusidae). Zootaxa, 4550: 579-584.

Campos, M.R.; Magalhães, C. and Rodríguez, G. 2003. The freshwater crabs of southern Colombia and their biogeographical affinities (Brachyura: Pseudothelphusidae). Nauplius (for 2002), 10: 15-25.

Cardona, L. and Campos, M.R. 2012. A new species of freshwater crabs of the genus Phallangothelphusa Pretzmann, 1965 from Colombia (Crustacea: Decapoda: Pseudothelphusidae). Zootaxa, 3515: 83-88.

Cumberlidge, N. 2016. Global Diversity and Conservation of Freshwater Crabs (Crustacea: Decapoda: Brachyura).p. 1-22. In: T. Kawai and N. Cumberlidge (eds), A Global Overview of the Conservation of Freshwater Decapod Crustaceans. Cham, Springer.

Cumberlidge, N.; Ng, P.K.L.; Yeo, D.C.J.; Naruse, T.; Meyer, K.S. and Esser, L.J. 2011. Diversity, endemism and conservation of the freshwater crab of China (Brachyura, Potamidae and Gecarcinucidae). Integrative Zoology, 6: 45-55.

Galvis, G. and Mojica, J.I. 2007. The Magdalena river freshwater fishes and fisheries. Aquatic Ecosystem Health \& Management, 10: 127-139.

IDEAM. 2013. Zonificación y codificación de unidades hidrográficas e hidrogeológicas de Colombia, Bogotá, http:// documentacion.ideam.gov.co/openbiblio/ bvirtual/022655/022655.htm. Accessed on 4 June 2020.

Izsák, J. and Papp, L. 2000. A link between ecological diversity indices and measures of biodiversity. Ecological Modelling, 130: $151-156$.

Magalhães, C. 1990. A new species of the genus Kingsleya from Amazonia, with a modified key for the Brazilian Pseudothelphusidae (Crustacea: Decapoda: Brachyura). Zoologische Mededelingen, 63: 275-281.

Magalhães, C. 2005. A new species of freshwater crab (Crustacea: Decapoda: Pseudothelphusidae) from the southeastern Amazon Basin. Nauplius, 12 (for 2004): 99-107.

Magalhães, C. 2009. A new species of freshwater crab of the genus Fredius Pretzmann, 1967 from the middle Amazon River basin, Brazil (Crustacea: Decapoda: Pseudothelphusidae). Proceedings of the Biological Society of Washington, 122: 81-86.

Magalhães, C. 2017. A new genus and species of freshwater crab (Decapoda: Pseudothelphusidae) from the Tapajós River, a southern tributary of the Amazon River in Brazil. Crustaceana, 90: 1015-1025.

Magalhães, C. and Pereira, G. 2007. Assessment of the decapod crustacean diversity in the Guayana Shield region aiming at conservation decisions. Biota Neotropica, 7: 1-14.

Magalhães, C. and Türkay, M. 2010. A new freshwater crab of the genus Brasiliothelphusa Magalhães and Türkay, 1986 from rio Aripuanã, southern Amazon region, Brazil. (Crustacea: Decapoda: Pseudothelphusidae). Nauplius, 18: 103-108.

Magalhães, C.; Sanches, V.Q.A.; Pileggi, L.G. and Mantelatto, F.L. 2014. Morphological and molecular description of a new species of Fredius (Decapoda: Pseudothelphusidae) from Rondônia, southern Amazonia, Brazil. p. 101-114. In: D.C.J. Yeo; N. Cumberlidge and S. Klaus (eds), Advances in Freshwater Decapod Systematics and Biology. Leiden, Brill. Crustaceana Monographs, 19. 
Magalhães, C.; Robles, R.; Souza-Carvalho, E.A.; Carvalho, F.L.; Oliveira Malta, J.C. de and Mantelatto, F.L. 2018. Annotated checklist of parasitic and decapod crustaceans from the middle and lower Xingu (Amazon Basin) above and below the Belo Monte dam complex, Pará State, Brazil. Proceedings of the Academy of Natural Sciences of Philadelphia, 166: 1-34

Magnusson, W.E.; Lima, A.L.; Luizão, R.; Luizão, F.; Costa, F.R.C.; Castilho, C.V. and Kinnup, V.F. 2005. RAPELD: a modification of the Gentry method for biodiversity surveys in long-term ecological research sites. Biota Neotropica, 5: 19-24.

Milne-Edwards, H. 1853. Mémoire sur la famille des Ocypodiens. Annales des Sciences Naturelle, Zoologie, 20: 163-228.

Pedraza, M. and Tavares, M. 2015. A new species of freshwater crab of the genus Kingsleya Ortmann, 1897 (Crustacea: Brachyura: Pseudothelphusidae) from Amazonia, Brazil. Zootaxa, 4032: 444-450.

Pedraza, M; Magalhães, C. and Tavares, M. 2016. A new genus of freshwater crab of the tribe Kingsleyini Bott, 1970 (Crustacea: Decapoda: Brachyura: Pseudothelphusidae) with description of a new species from Mato Grosso, Brazil. Zootaxa, 4173: 94-100.

Pereira, G.; Lasso, C.A.; Mora-Day, J.; Magalhães, C. and Campos, M.R. 2010. Crustáceos decápodos de la Orinoquia venezolana: biodiversidad, consideraciones biogeográficas y conservación. p. 357-365. In: C.A. Lasso; J.S. Usma; F. Trujillo and A. Rial (eds), Biodiversidad de la cuenca del Orinoco. Bases científicas para la identificación de áreas prioritarias para la conservación y uso sostenible de la biodiversidad. Instituto de Investigación de Recursos Biológicos Alexander von Humboldt, WWF Colombia, Fundación Omacha. Fundación La Salle de Ciencias Naturales e Instituto de Estudios de la Orinoquia (UNAL), Bogotá.

Pereira, G.; Lasso, C.A.; Mora-Day, J.; Magalhães, C.; Morales, M. and Campos, M.R. 2009. Lista de los crustáceos decápodos de la cuenca del río Orinoco (Colombia-Venezuela). Biota Colombiana, 10: 75-87.

Prahl, H. 1983. Hypolobocera gorgonensis sp. nov. (Crustacea: Brachyura: Pseudothelphusidae) un nuevo cangrejo de agua dulce de la Isla de Gorgona, Colombia. Cespedesia, 12: 105-110.

Prahl, H. 1987. Strengeriana antioquensis sp. nov. (Crustacea: Pseudothelphusidae) a new freshwater crab of Colombia. Caribbean Journal of Science, 23: 244-246.

Prahl, H. 1988. Fresh-water crabs (Crustacea: Decapoda: Pseudothelphusidae) of the Pacific drainage of Colombia. Zoologische Jahrbücher für Systematik, 115: 171-186.

Prahl, H. and Giraldo, J. 1985. Un nuevo cangrejo de agua dulce de la Cordillera Central de Colombia. Lozania, 49: 1-5.

Prahl, H. and Ramos, G. 1987. Potamocarcinus colombiensis sp. nov: Un nuevo cangrejo de agua dulce (Decapoda: Brachyura: Pseudothelphusidae) de la Serranía costera del Baudó, Colombia. Revista Biología Tropical, 35: 131-133.

Pretzmann, G. 1965. Vorläufiger Bericht über die Familie Pseudothelphusidae. Anzeiger der Österreichischen Akademie der Wissenschaften Mathematische Naturwissenschaftliche Klasse, (1) $1: 1-10$.

Pretzmann, G. 1967. Über eine Südamerikanische Süsswasserkrabben (Pseudothelphususidae). Vorläufige Mitteilung. Entomologisches Nachrichtenblatt, Wien, 14: 23-26.
Pretzmann, G. 1968. Neue Südamerikanische Süsswasserkrabben der Gattung Pseudothelphusa. Entomologisches Nachrichtenblatt, Wien, 15: 1-15.

Rahbek, C.; Borregaard, M.K.; Antonelli, A.; Colwell. R.K.; Holt, B.G.; Nogues-Bravo, D.; Rasmussen, C.M.Ø.; Richardson, K.; Rosing, M.T.; Whittaker, R.J. and Fjeldså, J. 2019. Building mountain biodiversity: Geological and evolutionary processes. Science, 365: 1114-1119.

Ramos-Tafur, G. 2006. Dos nuevas especies de cangrejos de agua dulce (Brachyura: Pseudothelphusidae) de la serranía de los Paraguas, Cordillera Occidental de Colombia. Revista Biología Tropical, 54: 1047-1056.

Ramos-Tafur, G. and Ríos, R. 2007. Hypolobocera olgaluciae, una nueva especie de cangrejo de agua dulce (Brachyura: Pseudothelphusidae) del río Chancos, departamento del Valle del Cauca, Colombia. Contribuciones al Estudio de los Crustáceos del Pacífico Este, 4: 39-45.

Rathbun, M.J. 1897. Descriptions de nouvelles especes de crabes d'eau douce appartenant aux collections du Muséum d'Histoire naturelle du Paris. Bulletin du Muséum nationale d'Histoire naturelle, Paris, 3: 58-61.

Rathbun, M.J. 1898. A contribution to a knowledge of the freshwater crabs of America. The Pseudothelphusidae. Proceedings of the United States National Museum, 21(1158): 507-537.

Rathbun, M.J. 1905. Les crabes d'eau douce (Potamonidae). Nouvelles Archives du Muséum d'Histoire Naturelle, Paris, 7: 159-321.

Rathbun, M.J. 1915. New freshwater crabs (Pseudothelphusa) from Colombia. Proceedings of the Biological Society of Washington, 28: 95-100.

Rodríguez, G. 1967. New species of Pseudothelphusidae from the Venezuelan Andes (Crustacea: Brachyura, Potamonidae). Zoologische Mededelingen, 42: 1-10.

Rodriguez, G. 1980. Description préliminaire de quelques especes et genres nouveaux de Crabes d'eau douce de l'Amérique tropicale (Crustacea: Decapoda: Pseudothelphusidae). Bulletin du Muséum nationale d'Histoire naturelle, Paris, (4) 2 Section A (3): 889-894.

Rodriguez, G. 1982. Les crabes d'eau douce d'Amérique. Famille des Pseudothelphusidae. Paris, ORSTOM, 223p. (Faune Tropicale, 22).

Rodríguez, G. 1985. A new cavernicolous crab (Crustacea: Decapoda: Pseudothelphusidae) from Colombia. Bioloski vestnik, Ljubljana, 33: 73-80.

Rodríguez, G. and Campos, M.R. 1989. Cladistic relationships of freshwater crabs of the tribe Strengerianini (Decapoda: Pseudothelphusidae) from the northern Andes, with comments on their Biogeography and descriptions of new species. Journal of Crustacean Biology, 9: 141-156.

Rodriguez, G. and Diaz. H. 1981. New species of freshwater crabs from the Andes (Crustacea: Decapoda: Pseudothelphusidae). Senckenbergiana biologica, 61: 305-312.

Rodríguez, G. and von Sternberg, R. 1998. A revision of the freshwater crabs of the family Pseudothelphusidae (Decapoda: Brachyura) from Ecuador. Proceedings of the Biological Society of Washington, 111: 110-139. 
Rodríguez, G. and Türkay, M. 1978. Der generische Status einiger Kolumbianischer Süsswasserkrabben. Senckenbergiana biologica, 59: 297-306.

Rodríguez, G. and Viloria A.L. 1992. Chaceus cesarensis a new species of the freshwater crab (Crustacea: Decapoda: Pseudothelphusidae) from Colombia with a key to the genus. Proceedings of the Biological Society of Washington, 105: 77-80.

Rodríguez, G.; Campos, M.R. and López, B. 2002. New species and records of pseudothelphusid crabs (Crustacea, Brachyura) from Colombia in the Tulane Natural History Museum. Tulane Studies in Zoology and Botany, 31: 1-17.

Schmitt, W.L. 1969. Colombian freshwater crab notes. Proceedings of the Biological Society of Washington, 82: 93-112.
Soberón, J.; Jiménez, R.; Golubov, J. and Koleff, P. 2007. Assessing completeness of biodiversity databases at different spatial scales. Ecography, 30: 152-160.

Suárez, H. 2015. Six new species of freshwater crabs from Pantepui, Venezuela (Crustacea: Decapoda: Pseudothelphusidae). Anartia, 25 (for 2013): 64-94.

Zanetti, F.; Macedo de Castro, P. and Magalhães, C. 2018. Freshwater crabs (Decapoda: Brachyura: Pseudothelphusidae, Trichodactylidae) from the state of Roraima, Brazil: species composition, distribution and new records. Nauplius, 26: e2018011.

Zimmer, C. 1912. Beitrag zur Kentniss der Süsswasser Dekapoden Kolumbiens. In: Fuhrmann, O. and E. Mayor, Voyage d'exploration scientifique en Colombie. Mémoires de la Société Neuchateloise des Sciences Naturelles, 5: 1-8. 